\title{
Un modelo de simulación computacional integrado a SIG para explorar la dinámica de crecimiento de la Zona Metropolitana de la Ciudad de México (1998-2008)
}

\author{
Marcos Valdivia López* \\ Nelly E. Linares Sánchez**
}

En este trabajo se explora la dinámica de crecimiento económico de los municipios y delegaciones de la Zona Metropolitana de la Ciudad de México (ZMCM) mediante simulaciones de un modelo dinámico de contagio tipo Autómata Celular integrado a Sistemas de Información Geográfica. Los resultados del modelo indican que en condiciones de intensa interacción local de los municipios o delegaciones, la ZMCM tiende a obtener una tasa de crecimiento promedio del PIB inferior a la que se ha observado en los últimos años. Además se muestra que la tasa de crecimiento en equilibrio de la ZMCM no se ve afectada por el carácter monocéntrico o policéntrico que pudiera presentar la metrópoli, pero sí por la cercanía o lejanía de los municipios o delegaciones con el centro o los subcentros económicos de la región. Finalmente, la investigación demuestra que es posible alcanzar tasas de crecimiento del PIB superiores a las observadas bajo ciertas condiciones de coordinación en la estructura urbana sin que necesariamente haya interacción global entre los municipios o delegaciones.

Palabras clave: interacción espacial, modelos computacionales, crecimiento económico local, Zona Metropolitana de la Ciudad de México.

Fecha de recepción: 15 de febrero de 2012.

Fecha de aceptación: 20 de septiembre de 2012.

A Computer Simulation Model Integrated into SIG to Explore the Dynamics of Growth of the Metropolitan Area of Mexico City (1998-2008)

This paper explores the dynamics of economic growth in the municipalities and delegations of the Metropolitan Area of Mexico City (MAMC) through simulations of a dynamic model of Cellular Automata contagion integrated into Geographic Information Systems. Model results indicate that under conditions of intense local interaction by municipalities and delegations, the MCMA tends to achieve a lower average growth rate

\footnotetext{
* Investigador del CRIM-UNAM. Correo electrónico: $<$ marcosv@correo.crim.unam.mx>.

** Posgrado en Economía de la FEs Acatlán-UNAM. Correo electrónico: <neeslisa@ yahoo.com.mx>.
}

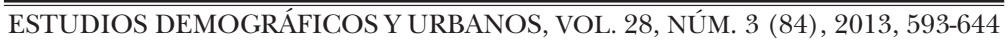


of GDP than has been observed in recent years. They also show that the equilibrium growth rate of the MCMA is not affected by the monocentric or polycentric nature of the metropolis, but rather by the proximity or distance of the municipalities and delegations in regard to the center or economic sub-centers of the region. Lastly, the research shows that it is possible to achieve higher GDP growth rates than those observed under certain conditions of coordination in the urban structure without necessarily being global interaction between municipalities and delegations.

Key words: spatial interaction, computer models, local economic growth, Mexico City Metropolitan Area.

\section{Introducción}

A pesar del notable crecimiento de las ciudades medias en décadas recientes, la Zona Metropolitana de la Ciudad de México (ZMCM) sigue concentrando gran parte de la actividad económica y poblacional del país; en 2008 los 76 municipios o delegaciones que la conforman generaron $31 \%$ del producto interno bruto (PIB) del país, y la región en su conjunto creció a una tasa anual promedio de 1.7\% (1998-2008). Sin embargo, al considerar la dinámica del PIB regional en el interior de la ZMCM encontramos que sus municipios y delegaciones crecen a tasas muy heterogéneas; por ejemplo, Isidro Fabela creció en el periodo mencionado a una tasa promedio de $27.1 \%$, mientras que el municipio de Cuautitlán decreció a una tasa de 5.3\%. ¿Qué factores explican este comportamiento tan heterogéneo en el interior de la ZMCM? ¿Es factible y adecuado utilizar los mismos modelos regionales que analizan el crecimiento entre regiones (estados) del país (por ejemplo los modelos tradicionales de convergencia o divergencia) para estudiar el crecimiento de unidades regionales en el interior de una zona metropolitana como la de la Ciudad de México?, ¿ conviene utilizar enfoques regionales que prioricen otro tipo de mecanismos en el interior de las ciudades? Cabe advertir al respecto que los enfoques teórico regionales recientes que analizan el crecimiento de las ciudades tienden a privilegiar el estudio de la interacción local (espacial) de los componentes microrregionales que conforman una ciudad o zona metropolitana; para poner esto en perspectiva en la polémica sobre modelación regional se ha dejado de analizar las ciudades como sistemas que se organizan de arriba hacia abajo para dar paso a enfoques de abajo hacia arriba (Batty, 2011). 
En años recientes el estudio de la interacción espacial entre microrregiones en el interior de áreas urbanas ha derivado en el desarrollo de modelos computacionales regionales (muchos de ellos basados en el Autómata Celular), y en particular ha ganado popularidad un área de estudio que integra dichos modelos con Sistemas de Información Geográfica (SIG) debido a la potencialidad para mejorar la incorporación de patrones espaciales y procesos temporales reales (Brown et al., 2005). Aunque recientemente el número de aplicaciones que integran enfoques computacionales y SIG se ha incrementado considerablemente, la metodología e integración de ambas técnicas se encuentra todavía en fase de desarrollo (Crooks et al., 2008); sin embargo con el uso de dichas técnicas se pueden generar modelos computacionales más útiles y realistas, ya que están abocados a comprender las dinámicas regionales que se dan en un contexto histórico regional específico, y a facilitar con ello el diseño de políticas regionales. Para el caso de México estos enfoques todavía no se han explorado suficientemente (Manson, 2004) y según nuestro conocimiento no hay antecedente alguno de su uso para investigar dinámicas de crecimiento económico regional.

En este trabajo adoptamos un enfoque bottom-up al estudiar un modelo simple de contagio entre unidades regionales integrado al Sistema de Información Geográfica (SIG) de la ZMCM, con el propósito de destacar ciertos patrones de crecimiento en el interior del área metropolitana que están condicionados únicamente por la estructura y el arreglo espacial de sus municipios o delegaciones. En particular la investigación pone énfasis en la manera en que el mecanismo de contagio (visto éste como una externalidad no pecuniaria que afecta el crecimiento en la ciudad) opera bajo diferentes escenarios de actualización y arranque del sistema, y también se interesa en cómo se ve afectado cuando se introduce una estructura policéntrica en el interior de la ZMCM. En general encontramos que al analizar el crecimiento de la ZMCM bajo un modelo de contagio se observa que la concentración y la dependencia espacial del crecimiento municipal o delegacional genera una tasa de equilibrio inferior a la observada en años recientes; de igual forma los resultados de las simulaciones indican que la tasa de equilibrio promedio del crecimiento no se altera si se asume una estructura monocéntrica o policéntrica de la ZMCM, pero la cercanía o lejanía respecto al centro o los subcentros sí es importante en la determinación de la tasa de crecimiento global.

El trabajo está integrado por cinco secciones. En la primera se aborda someramente el enfoque computacional en el marco de los 
modelos de interacción espacial que estudian el crecimiento regional. En la segunda sección se presenta información sobre el análisis de la estructura urbana de la ZMCM y su dinámica de crecimiento durante los últimos 15 años. La tercera sección muestra el modelo de contagio propuesto, sus características y su integración al SIG de la ZMCM. En la cuarta sección se refieren los experimentos y resultados de la simulación del modelo, y finalmente se concluye con algunos comentarios finales.

\section{El enfoque computacional y su integración en escenarios regionales reales}

En las teorías recientes sobre el crecimiento regional ha sido relevante el análisis de las interacciones interregionales de las economías locales dentro de un sistema agregado (llámese región, país o continente). Conforme a estos nuevos enfoques se argumenta que el contexto geográfico o espacial de una economía local influye directamente sobre las oportunidades de crecimiento de ésta y de la región (Capello y Nijkamp, 2009). De manera que en las economías locales puede haber importantes dinámicas de interacción local que influyan en el crecimiento económico del sistema o en el del conjunto de las regiones. Según Glickman (1982) los efectos se pueden ver en dos sentidos: i) tipo bottom-up: de las economías locales (y de su interacción) hacia arriba (del sistema), y ii) tipo top-down, de arriba (del sistema) hacia abajo (economías locales).

El crecimiento económico regional que se aborda a partir de este tipo de impactos (bottom-upy top-down) no sólo se explica por dinámicas de interacción regional-espacial, también incluye otros mecanismos causales y dinámicos de tipo competitivo, institucional, geográfico, territorial y endógeno, de tal suerte que la investigación del fenómeno del crecimiento regional se torna más compleja. Frente a esto los modelos computacionales, en especial los modelos basados en agentes (MBA) y su integración a los SIG ofrecen una alternativa metodológicamente viable para implementar modelos regionales complejos como los ya mencionados. En particular los MBA son una herramienta relativamente nueva en las ciencias sociales y con su uso se han extendido las posibilidades de la modelación tradicional al integrar procesos dinámicos en el análisis, y poder estudiar las propiedades emergentes y las microdiversidades (Crooks et al., 2008). 
Desde una perspectiva de modelación regional un modelo computacional puede definirse como el conjunto de reglas que gobiernan el comportamiento dinámico de múltiples agentes (regiones) que componen un sistema (regional). Las reglas de modelación rigen el estado de cada agente (por ejemplo, el PIB de una región). En particular, el estado de un agente (región) puede depender de la interacción e interdependencia de los propios agentes (regiones), así como de la interacción con el medio o contexto. La modelación de la interacción entre agentes (regiones) "desde abajo" conlleva la formación de un resultado global emergente ${ }^{1}$ del sistema (regional) que se está modelando (por ejemplo, la dinámica del PIB de la región).

Son varias las aproximaciones metodológicas que pueden emplearse para modelar con perspectiva computacional, y cada una tiene características particulares; entre ellas destacan los Autómatas Celulares (AC), los modelos basados en agentes, los modelos de aprendizaje o evolutivos, etc. En particular los AC son un modelo matemático que sirve para analizar sistemas dinámicos espaciales de tipo discreto; se pueden analizar fácilmente con una perspectiva computacional y son muy útiles para estudiar los sistemas regionales. Un AC está compuesto por un conjunto de celdas ordenadas en un espacio regular de dimensión $D$; generalmente es una cuadrícula de dos dimensiones, a la manera de un tablero de ajedrez. Se incluye un número finito de estados y una regla de transición que se aplica a todas las celdas. El tiempo se mide de forma discreta, por lo que en cada periodo se actualizan las celdas de manera simultánea o secuencial y la regla de transición permite calcular el estado de cada celda a partir de reglas locales. La regla local puede definir el estado presente de una celda a partir de su estado previo, del estado previo de sus vecinas o de ambos (Hagselmann y Flache, 1998).

Los AC clásicos han sido utilizados ampliamente en las ciencias sociales para analizar diversos fenómenos, como el crecimiento urbano, el uso de suelo, la segregación residencial, el tráfico, etc. Por ejemplo, en economía un AC tiene su principal asociación con la obra de Schelling (1978) y su modelo de segregación. Los AC se han considerado particularmente en los nuevos modelos de economía urbana de cam-

1 De acuerdo con Gilbert y Troitzsch (2006: 11) "la emergencia ocurre cuando las interacciones sobre los objetos en un nivel dan lugar a tipos diferentes de objetos en otro nivel. En otras palabras, un fenómeno es emergente si requiere de categorías nuevas para describirlo, que no son para describir el comportamiento de sus componentes subyacentes. Las simulaciones pueden suministrar una poderosa metáfora para tales investigaciones teóricas". 
bio del suelo; Irwin (2010) ha observado al respecto que una tercera parte de los 100 artículos más relevantes en esta área desde 2003 modelan conforme a esta perspectiva, y la situación no parece muy distinta en los últimos desarrollos modelísticos que se han elaborado en el campo del desarrollo regional (véase la Introducción, en Capello y Nijkamp, 2009).

Sin embargo los AC se basan en una estructura de interacción espacial homogénea, ya que sus celdas son regulares, de ahí que en años recientes se considerara la necesidad de incorporar la heterogeneidad espacial en un AC emulando ambientes realistas por medio de celdas irregulares. Flache y Hagselmann (2001) mostraron que aunque los resultados de los modelos tipo AC con una estructura irregular son también robustos respecto a los tradicionales, el uso de una estructura irregular puede ampliar o retardar los efectos espaciales que un AC tradicional comúnmente genera. Page (1999: 186) llegó a una conclusión similar al proponer un modelo de formación de ciudades bajo una estructura tipo AC de celdas regulares y advertir que los resultados analíticos podrían verse afectados si se consideraran celdas irregulares.

Asimismo se ha desarrollado una alternativa de modelación para incluir "estructuras irregulares" en modelos como el AC (o tipo ABM): la de integrarlos con SIG. Conviene subrayar aquí que los SIG son una plataforma muy potente para el análisis de la información geoespacial, y han sido muy útiles para representar los insumos (o variables) y los resultados de un modelo con naturaleza geoespacial; sin embargo los SIG por sí solos no han sido lo más adecuado para modelar sistemas dinámicos con carácter geoespacial, y en particular la representación del tiempo en estos intentos ha generado muchos problemas (Brown et al., 2005). De esta manera, los SIG también han encontrado un terreno de modelación dinámica robusta al incorporarlos a los modelos tradicionales espaciales tipo AC y MBA (Crooks et al., 2008).

Hoy día puede consultarse una literatura relativamente amplia sobre la integración; por ejemplo el trabajo de Moreno et al. (2009) para un examen puntual del AC y su transformación geométrica en objetos, así como la reciente compilación de Heppenstall et al. (2012) sobre diversos estudios que han integrado ABM con SIG. En las siguientes secciones retomaremos algunos elementos metodológicos de esta discusión y los consideraremos en la construcción de un modelo simple de contagio para analizar el crecimiento regional de la Zona Metropolitana de la Ciudad de México. 


\section{La ZMCM y su dinámica de crecimiento (1998-2008)}

Uno de los elementos centrales de los estudios contemporáneos sobre la estructura urbana de la Ciudad de México es la determinación de si su carácter es mono o policéntrico. Los modelos teóricos asociados a este debate son: el de círculos concéntricos (Park y Burgess, 1925), los modelos monocéntricos tipo Alonso-Muth-Mills, ${ }^{2}$ y los de núcleos múltiples o policéntricos. ${ }^{3}$ Para el caso mexicano la mayoría de los estudios caracteriza a la Ciudad de México como policéntrica, pero el debate dista de estar cerrado. ${ }^{4}$ En particular su estructura urbana se ha analizado valiéndose de indicadores como el uso de suelo, la densidad de la población y el empleo (Aguilar y Alvarado, 2004; Suárez y Delgado, 2009), la segregación (Escamilla, 2006; Saraví, 2008) y la movilidad intraurbana de tipo residencial y laboral (Graizbord y Acuña, 2004, 2006 y 2007; Sobrino, 2006; Suárez y Delgado, 2010), entre otros. Sin embargo, en los estudios urbanos sobre México no se ha puesto especial atención en otra perspectiva: la relación entre la concentración urbana y el desarrollo económico local. ${ }^{5}$ La concentración urbana genera por sí misma ventajas como la proximidad espacial, el ahorro en el costo de transporte, mercados más eficientes y rendimientos crecientes que influyen positivamente en la productividad y en el crecimiento y el desarrollo económicos (Quigley, 1998; Henderson, 2003). Como veremos más adelante en la cuarta sección, en este estudio emprendimos una primera aproximación al respecto al proponer un modelo de interacción espacial (que incluye además subcentros económicos) para explicar el crecimiento local de los municipios de la ZMCM.

${ }^{2}$ En un modelo monocéntrico tipo Alonso-Muth-Mills, un "centro económico" estructura la actividad urbana de una ciudad. Este modelo postula un concepto de equilibrio espacial en donde el flujo de elementos que determinan la localización residencial (ingreso, precios de vivienda, amenidades y costos de transporte) permanece constante sobre el espacio. Una predicción central de este modelo es que los precios de la vivienda declinan cuando las amenidades y el ingreso son constantes, conforme los costos de transporte aumentan con la distancia al centro económico (Glaeser, 2008).

${ }^{3}$ En estos modelos la actividad urbana se estructura en subcentros. En general la literatura respectiva tiene dos vertientes: una empírica orientada a la localización de los subcentros (usando indicadores como concentración del empleo) y otra más teórica que examina las condiciones en que surge el policentrismo (Richardson, 1988).

${ }^{4}$ Por ejemplo, Graizbord y Acuña (2004) y Aguilar y Alvarado (2004) sostienen que la Ciudad de México es policéntrica, mientras que Suárez y Delgado (2009) aseguran que está en transición de una estructura monocéntrica hacia una policéntrica.

5 Suárez y Delgado (2009 y 2010) sugieren que la relación entre la estructura urbana y el crecimiento económico es uno de los temas que conviene desarrollar en los estudios futuros de estructura urbana en México. 
Administrativamente la ZMCM está conformada por 16 delegaciones del Distrito Federal, 59 municipios del Estado de México y uno de Hidalgo (Gaceta Oficial del Distrito Federal, 23 de enero de 2006). Su representación territorial se muestra en el mapa 1.

Según las estimaciones que utilizamos en esta investigación, ${ }^{6}$ el PIB de la ZMCM representa poco más de $30 \%$ del PIB nacional, pero tal participación ha venido disminuyendo paulatinamente en las últimas décadas; ha pasado de 34\% en 1998 a 31\% en 2008 (véase el cuadro 1). El crecimiento del PIB de la ZMCM ha sido menos dinámico que el del conjunto del país: en el periodo 1998-2008 la ZMCM creció a una tasa promedio anual de $1.7 \%$, mientras que el país lo hizo a un ritmo de 2.6 por ciento.

En el interior de la ZMCM el PIB de los municipios y delegaciones creció a una tasa promedio de $5.7 \%$ en el periodo 1998-2008; pero este promedio oculta la fuerte heterogeneidad de tal crecimiento entre las microrregiones, pues mientras las primeras cinco mayores tasas promediaron anualmente 20\% (Isidro Fabela, Atlautla, Valle de Chalco, Jaltengo y Jilotzingo), las últimas cinco muestran un decrecimiento promedio anual de $4.1 \%$ (Cuautitlán, Azcapotzalco, Milpa Alta, Gustavo A. Madero y Tepetlaoxtoc). Las mayores tasas de crecimiento que se registraron en el periodo 1998-2008 coinciden con los municipios más pobres en 1998, de ahí que dichas regiones mejoraran notablemente su condición económica para el año 2008; esto se puede observar en la gráfica 1, donde se despliega el diagrama de dispersión entre el Log del PIB per cápita 1998 (en el eje de las X) y el crecimiento en el periodo 1998-2008 del Log del PIB per cápita.

En realidad la gráfica 1 muestra lo que se conoce en la literatura como convergencia absoluta; y la línea de tendencia es la regresión beta cuyo coeficiente es negativo y significativo, con lo que se sugiere una dinámica de convergencia entre los municipios y delegaciones de la ZMCM. (Si se asume un modelo neoclásico a la Barro, la regresión beta estimada sugiere una velocidad de convergencia de 0.022 , es decir, los municipios y delegaciones convergen hacia su estado estacionario del PIB per cápita a una tasa de $2.2 \%$ anual).

${ }^{6}$ Esta investigación utiliza estimaciones municipales y delegacionales basadas en una metodología que elaboraron miembros del posgrado de Economía de la UNAM y que utiliza información del PIB regional y de los censos económicos del INEGI. Véase el "Modelo de Impacto Regional en México" (2012), proyecto a cargo del profesor investigador Miguel Ángel Mendoza. 
MAPA 1

Representación geográfica de la ZMCM

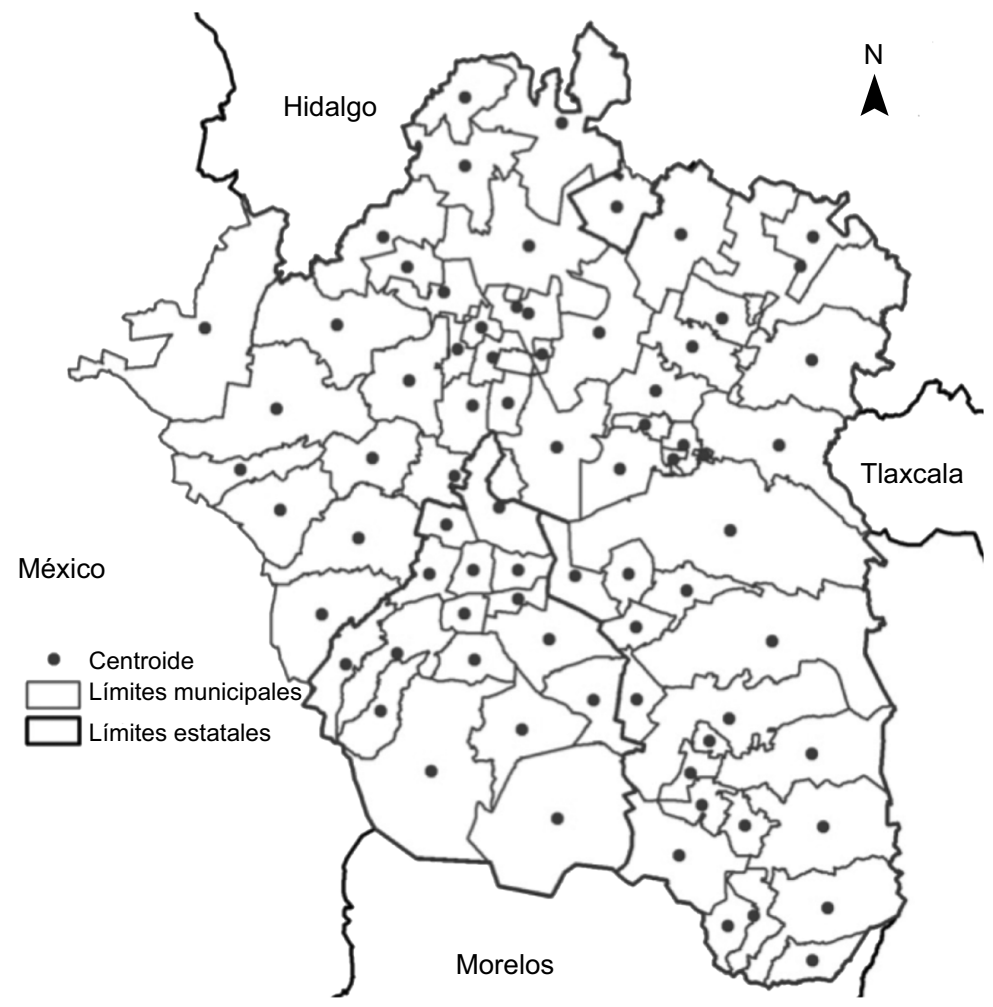

FUENTE: Marco geoestadístico municipal 2005, versión 1.0.

Si bien esto indica que los municipios y delegaciones más pobres están creciendo a tasas más aceleradas que sus contrapartes "más ricos", también es cierto que las delegaciones del surponiente de la ZMCM que ya ocupaban una posición relativamente privilegiada en 1998 (como es el caso de Cuajimalpa y Álvaro Obregón) crecieron a mayores tasas en su PIB per cápita, mientras que otras del suroriente de la ZMCM (las "menos desarrolladas" del Distrito Federal) sufrieron incluso decrecimientos (tal es el caso de Venustiano Carranza, Iztapalapa, Gustavo A. Madero y Milpa Alta). Además, como veremos más adelan- 


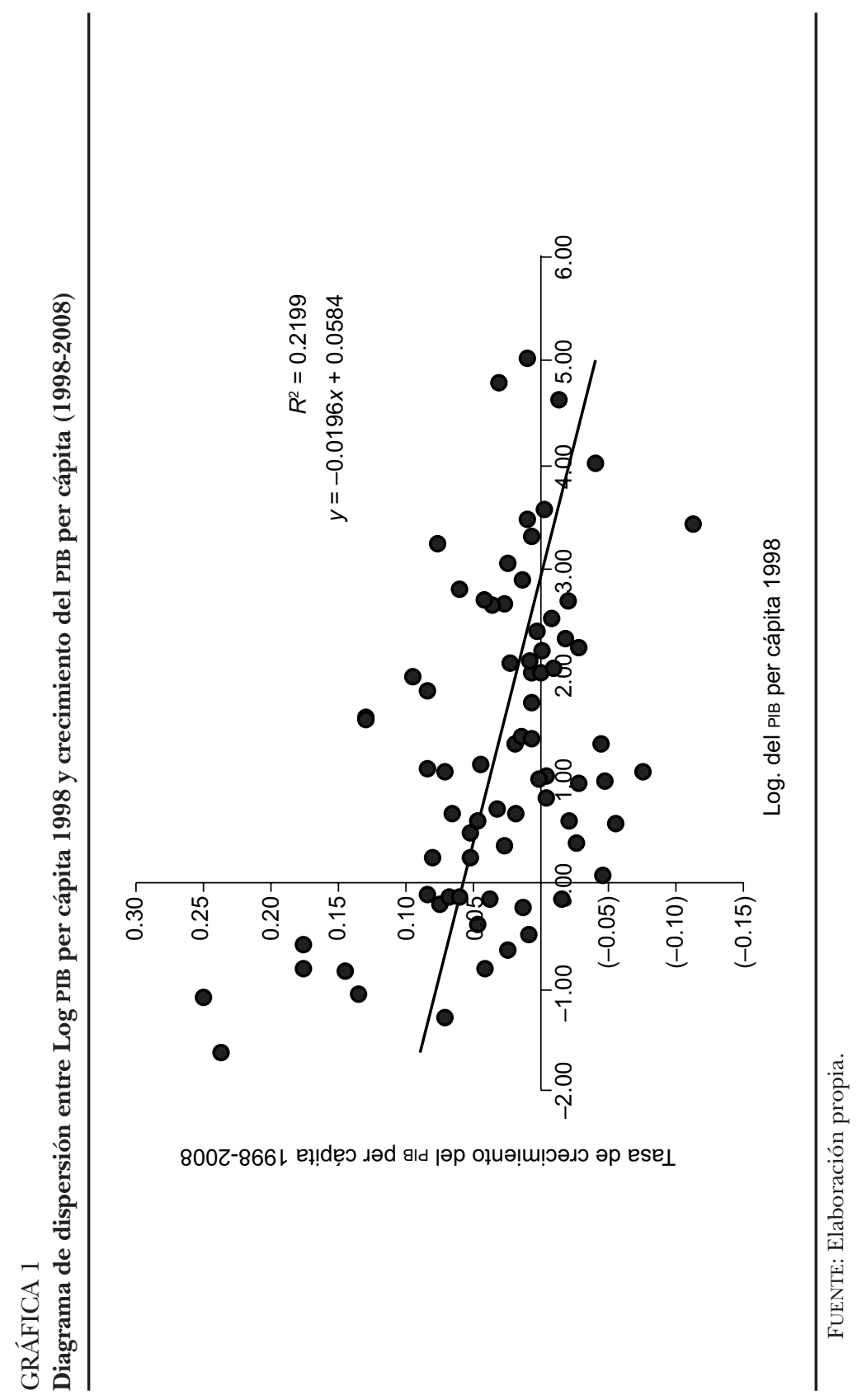


Valdivia y Linares, UN MODELO DE SIMULACIÓN

CUADRO 1

Participación del PIB y crecimiento de la ZMCM y del DF en México (1998-2008)

\begin{tabular}{lccc}
\hline \multicolumn{4}{c}{ PIB (miles de pesos a precios de 1993) } \\
\hline & Nacional & DF & ZMCM \\
\hline Núm. obs. & 1 & 16 & 76 \\
1998 & $100 \%$ & $25 \%$ & $34 \%$ \\
2003 & $100 \%$ & $24 \%$ & $32 \%$ \\
2008 & $100 \%$ & $23 \%$ & $31 \%$ \\
\hline & $1998-2008$ & & \\
\hline Crecimiento periodo & $28.9 \%$ & $18.5 \%$ & $18.5 \%$ \\
Crecimiento promedio anual & $2.6 \%$ & $1.7 \%$ & $1.7 \%$ \\
Media delegaciones-municipios & - & $1.9 \%$ & $5.7 \%$ \\
d.e. & - & 0.05 & 0.07 \\
Coef. de variación 1998-2008 & - & 2.43 & 1.19 \\
\hline
\end{tabular}

FUENTE: Elaboración propia con base en datos de INEGI y estimaciones realizadas del PIB municipal-regional (véase la nota 6).

te, los municipios y delegaciones de la ZMCM despliegan una dependencia espacial en su crecimiento económico (véase el cuadro 4, columnas 2 y 3 ), lo que lleva a cuestionar los modelos tipo convergencia, como el aludido, para entender la dinámica de crecimiento de la ZMCM, ya que esencialmente asume que hay independencia de las unidades de observación regional. ${ }^{7}$

Por lo expuesto, la dinámica de crecimiento en el interior de la ZMCM se observa más compleja de lo que los modelos tradicionales de crecimiento, diseñados para una escala regional superior, pudieran aportar al análisis. Si bien la ZMCM en su conjunto está creciendo a un ritmo menor que el del conjunto del país, este dato promedio oculta y está lejos de reflejar una dinámica heterogénea y cambiante entre los municipios y delegaciones de la ZMCM.

Con la intención de avanzar en esta dirección, en las siguientes secciones analizaremos la dinámica de crecimiento de la ZMCM por

${ }^{7}$ Según Capello y Fratesi (2008: 49) hay dos enfoques en el análisis empírico del crecimiento regional: el "a-espacial" donde el espacio se analiza considerando las características propias de la región y dejando fuera cualquier interacción económica, social y física con otras áreas; y el "espacial", que analiza las regiones como parte de un sistema y por lo tanto trata de capturar los efectos de la interacción entre regiones. 
medio de un modelo de contagio (que en sí es un modelo de convergencia) que, a diferencia de los modelos tradicionales, toma en cuenta la interdependencia y la interacción espacial de las delegaciones y los municipios de la región.

\section{Modelo y estructura de simulación}

El modelo que se presenta en esta sección se diseñó para estudiar el comportamiento dinámico de la tasa de crecimiento del PIB en la ZMCM. $\mathrm{Su}$ lógica se basa en el cálculo de tasas de crecimiento municipal a partir de una regla de interacción local en que se "adopta" la tasa de crecimiento de los "vecinos cercanos", definidos éstos con un criterio de contigüidad y por radios de distancia.

En cualquier tiempo $t$ la economía de la ZMCM consiste de $N=76$ municipios o delegaciones. ${ }^{8}$ Cada municipio-delegación $i \in N$ crece en el tiempo $t$ a una tasa $G r^{t}$; y está localizada en puntos del plano cartesiano $x, y$ [que denotan las coordenadas y ubicación $q_{i}$ del centroide de la unidad administrativa delegacional o municipal], tal que $(x, y) \in X$, donde $X$ es el espacio en que están localizados los municipiosdelegaciones $i$ [X puede verse como el mapa o la cartografía de la ZMCM; véase el mapa 1]. $X=D^{2}$, ya que estamos considerando un espacio en dos dimensiones. El estado de los municipios y delegaciones está definido por un vector $U$ cuyos componentes son la tasa de crecimiento $G r_{i}$ y la localización de las unidades regionales $q_{i}$. Una representación formal de un sistema dinámico discreto de la ZMCM con los anteriores elementos es:

$$
G r_{i}^{t+1}=u_{i, t}\left(G r_{1}^{t}, \ldots, G r_{76}^{t}, q_{i, 1}, \ldots, q_{i, 76}\right)
$$

Formalmente la relación general anterior obtiene la siguiente expresión particular para el modelo de contagio que analizamos en este trabajo y que considera una distancia $R$ de interacción espacial:

$$
G r_{i}^{t+1}=\beta_{g} \frac{1}{n_{i}} \sum G r_{j \neq i \mid t j-i \| \leq R}^{t} \quad j=1,2 \ldots 76
$$

${ }^{8}$ El municipio-delegación se concibe como un agente en el sentido que representa una unidad autocontenida con características particulares (i.e. tasa de crecimiento y posición geográfica relativa), y a partir de su interacción con otros (agentes-municipios) su contexto espacial puede cambiar de estado en el sistema (véase Castañeda, 2009: 12). 
donde Gr es la tasa de crecimiento de $i$ (municipio o delegación de la ZMCM), $n_{i}$ es el número de municipios o delegaciones vecinas $j$ que están localizados dentro de un radio de distancia euclidiana $R$ desde $i$; la distancia se toma desde el centroide del polígono de la delegación o municipio; y finalmente $\beta$ es un parámetro de intensidad de interacción espacial o de imitación del crecimiento de los vecinos.

De igual manera, el modelo tiene la siguiente expresión (en forma matricial) cuando se consideran sólo los vecinos contiguos de $i$ (o tipo queen):

$$
G r^{t+1}=\beta_{g} W G r^{t}
$$

donde $\boldsymbol{G} \boldsymbol{r}$ es un vector $(n \times 1)$ de tasas de crecimiento, $\boldsymbol{W}$ es una matriz de interacción espacial $(n \times n)$, y sus elementos tienen la característica $w_{i j}=0$ si los municipios $i$ y $j$ no son contiguos, y $w_{i j}=1 / a_{i}$ si $i$ y $j$ son contiguos donde $a_{i}$ es el número de municipios o delegaciones contiguas de $i$, de tal forma que cada elemento de $W$ sea estandarizado por renglón, es decir, $\Sigma_{j} w_{i j}=1$.

Las ecuaciones anteriores se pueden interpretar como la versión continua determinística del Voter Model que desarrolló Liggett (1985) en el campo de la física estadística. El resultado central a destacar es la dinámica de contagio que genera este tipo de modelos de interacción espacial, donde $\beta$ representa el parámetro que mide la influencia de los vecinos $j$ en el crecimiento del municipio $i$. Como ya se expuso, esta "influencia" en el modelo representa la tasa de crecimiento promedio de los vecinos del municipio $i$ en el tiempo $t$ acotada por el tipo de vecindad aplicado.

Para fines de monitoreo del comportamiento agregado de la ZMCM se considera un promedio simple global del crecimiento de los municipios o delegaciones en cada ciclo como una agregación de los microestados: ${ }^{9}$

${ }^{9}$ La agregación de tasas de crecimiento a partir de regiones "arbitrarias" puede llevarnos a inferencias erróneas sobre el comportamiento individual; en particular, el dato agregado de crecimiento puede tener un problema de falacia ecológica o un problema de la unidad de área modificable (MUAP en inglés), ambos relacionados con la heterogeneidad espacial. La partición regional en este estudio fue escogida porque es la unidad administrativa más desagregada disponible que ofrece información confiable del PIB, pero esto no garantiza que sea la mejor partición funcional para considerar la generación del valor agregado. Una forma tradicional de evitar el sesgo estadístico debido a la partición escogida consiste en usar un promedio ponderado de las tasas de crecimiento individuales (véase Hwa, 2011), siendo el ponderador, para el caso de 


$$
G r^{t+1^{*}}=\frac{1}{76} \sum_{i=1}^{76} G r_{i}^{t+1}
$$

Con las ecuaciones anteriores manejaremos una combinación de los siguientes escenarios de simulación: $i$ ) orden de ejecución: asincrónico o sincrónico, ii) determinados umbrales de visión para interactuar, y iii) procesos de interacción ordenados territorialmente -de las localidades más lejanas o de las localidades más cercanas- hacia el centro económico de la ciudad.

Las unidades regionales modeladas son heterogéneas por varias razones: i) por su ubicación geográfica, la cual les imprime automáticamente una característica espacial particular; ii) por su vecindario; y iii) por su tasa de crecimiento, la cual corresponde a la tasa observada y condensa las características económicas propias de cada municipio. Estos elementos permiten introducir la heterogeneidad en las ecuaciones 1 y 1 .A, y consecuentemente modelar el crecimiento económico como un proceso de retroalimentación continua entre municipios y delegaciones heterogéneos, de tal suerte que el crecimiento de la ZMCM se entiende como el resultado de la interacción de las partes y no como una simple adición de la producción que se genera en cada unidad regional.

nuestro estudio, la participación de cada municipio o delegación en el PIB agregado. Otra forma de evitar el sesgo es asignando un parámetro beta individual a cada unidad espacial, es decir, añadiendo un subíndice $i$ al coeficiente beta de la ecuación [1] y [1.A]; en la literatura econométrica una aproximación semejante puede darse estimando la ecuación de crecimiento mediante regresiones ponderadas geográficamente (Peeters y Chasco, 2006: 260) o usando estimaciones locales autorregresivas espaciales (Ertur y Koch, 2007). El camino que escogimos en este trabajo, para considerar el efecto de partición, fue simplemente incluir un factor de ponderación a partir de considerar el PIB per cápita (usando como denominador la población total) y el valor agregado censal bruto por trabajador ocupado (véase la cuarta sección). Adicionalmente debe señalarse que el impacto de las externalidades espaciales sobre el crecimiento puede verse también afectado por la partición escogida; y si bien este aspecto todavía ha sido poco abordado en la literatura, debe tomarse en cuenta. Al respecto Resende (2011) evalúa el crecimiento económico y el efecto de las externalidades espaciales en diferentes escalas regionales (estados, municipios, microrregiones y aglomeraciones espaciales) para el caso de Brasil, y entre sus hallazgos destaca que las externalidades espaciales sólo aparecen en las escalas pequeñas (municipales y microrregionales). En el presente estudio estamos considerando una unidad de partición relativamente pequeña (municipio), por lo que la presencia de externalidades en las ecuaciones (A) y (A.1) es en principio factible. 


\section{Valdivia y Linares, UN MODELO DE SIMULACIÓN}

\section{La simulación}

El proceso de simulación que se propone para estudiar las ecuaciones 1 y 1 .A se sintetiza en tres pasos: $:^{10}$ en el primero se integra el SIG a la interfaz del programa de simulación; en el segundo se asigna un criterio de vecindad (tipo queen o el criterio de radios de distancia), y en el tercero se ejecuta la regla de transición (ecuaciones 1 y 1.A). Debido a que estamos simulando la dinámica de ecuaciones discretas, la simulación calculará en cada instante de tiempo $(\text { tick })^{11}$ una tasa de crecimiento individual y a su vez contabilizará un dato global, el cual expresará el crecimiento promedio de las regiones de la ZMCM (véase la ecuación 2). En el esquema 1 se describen visualmente los pasos de simulación mencionados.

\section{La estructura espacial de interacción}

En un modelo cuyo eje central es la interacción espacial se tiene que definir una estructura espacial de interacción o tipo de vecindad para los agentes que se modelan. En un AC de tipo clásico la vecindad es homogénea y simétrica; puede ser de tipo Moore, Von Newman o generarse al definir radios de influencia. En contraste, cuando la distribución espacial de los agentes (o unidades regionales) está acotada por una representación cartográfica, la distribución de los mismos ya no es simétrica y por consecuencia el tamaño de los vecindarios deja de ser homogéneo. En este caso las vecindades tipo Moore o Von Newman tienen que adaptarse a la estructura no simétrica de las unidades espaciales que tiende a desplegar la mayoría de las cartografías.

Para los ejercicios de simulación se construyeron dos estructuras espaciales de interacción. El primer tipo de vecindad lo denominamos queen y está definido como una matriz de pesos espaciales, tal como se

${ }^{10}$ La plataforma de software que se empleó para la construcción y análisis del modelo fue NetLogo (versión 4.1.3), autorizado por Uri Wilensky en $2011<\mathrm{http}$ // ccl. northwestern.edu/netlogo>, Center for Connected Learning and Computer-Based Modeling, Northwestern University, Evanston, IL. Este paquete incluye una extensión GIS que permite desplegar información vectorial e información tipo Raster. La ventaja de ello es que podemos construir un ambiente de simulación donde es posible desplegar un mapa que contiene información georreferenciada de la ZMCM.

11 A lo largo de este trabajo los términos "instante de tiempo" y "tick"se utilizan de manera indistinta. Una corrida se define como el conjunto de instantes de tiempo o ticks en los cuales el proceso bajo estudio se estabiliza o llega al equilibrio. 


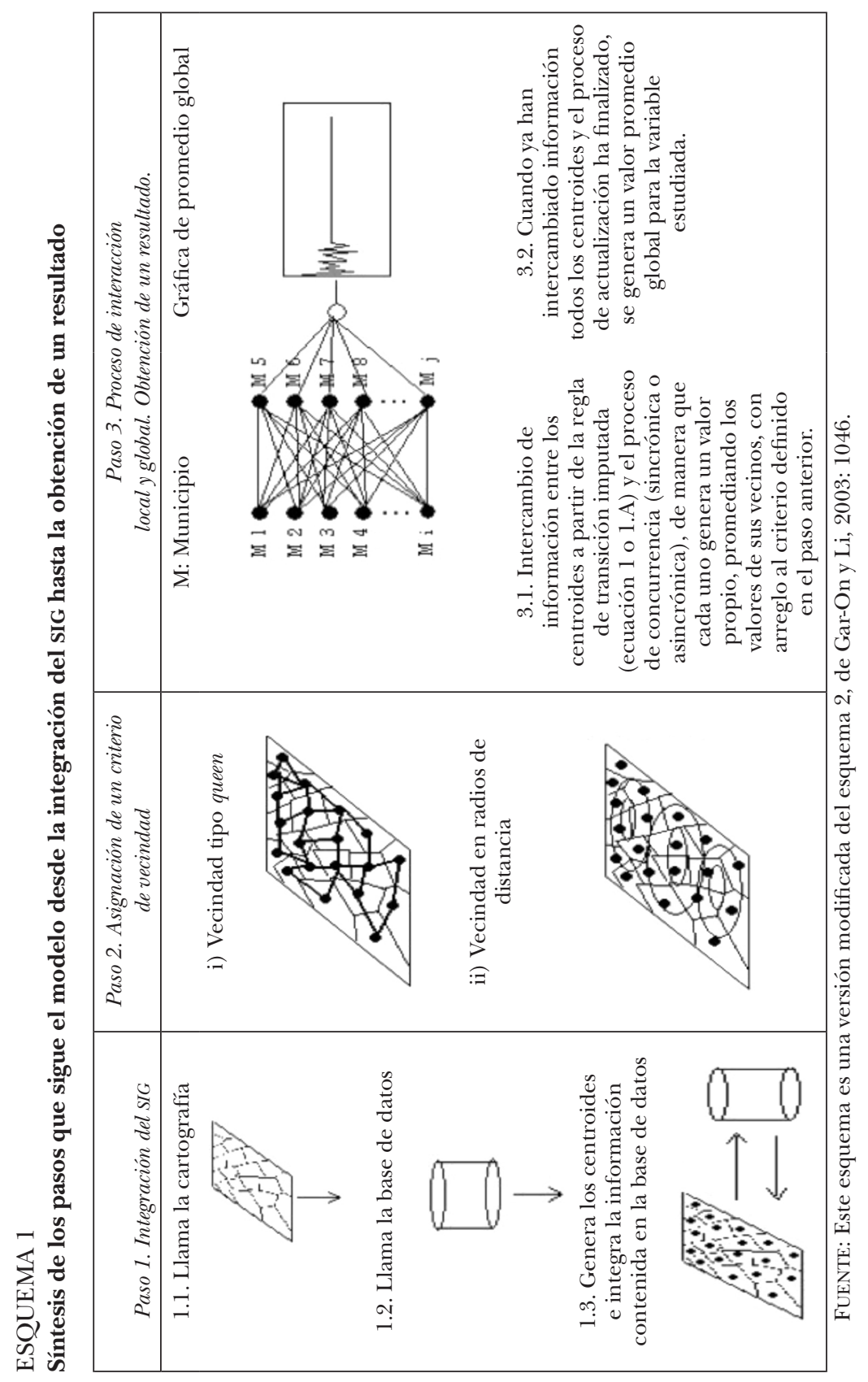


expresa en la ecuación 1.A. En términos cartográficos, los vecinos con los cuales un polígono generará un vínculo de retroalimentación serán aquellos otros con los que comparta aristas y vértices. La gráfica 2 ilustra el histograma de frecuencias del número de vecinos que tiene cada municipio o delegación de la ZMCM conforme a un vecindario tipo queen. Según el histograma el número de vecinos bajo esta modalidad de interacción es heterogéneo, y en promedio cada municipiodelegación interactúa con cinco municipios. Los casos extremos son Ecatzingo y Nopaltepec, con sólo un vecino; y Texcoco, que por su posición geográfica relativa tiene 10 municipios vecinos.

La segunda estructura espacial de interacción considera vecinos de $i$ todos aquellos municipios $j$ que caen dentro del radio de influencia de $i$, tal como se define en la ecuación 1 . La vecindad tipo radio consiste en ubicar áreas de influencia donde el centroide de cada municipio o delegación funge como el centro de un círculo, mientras que la distancia es el radio de dicha área de influencia (véase la $R$ en

\section{GRÁFICA 2}

Distribución de los municipios y delegaciones de acuerdo a un vecindario tipo queen

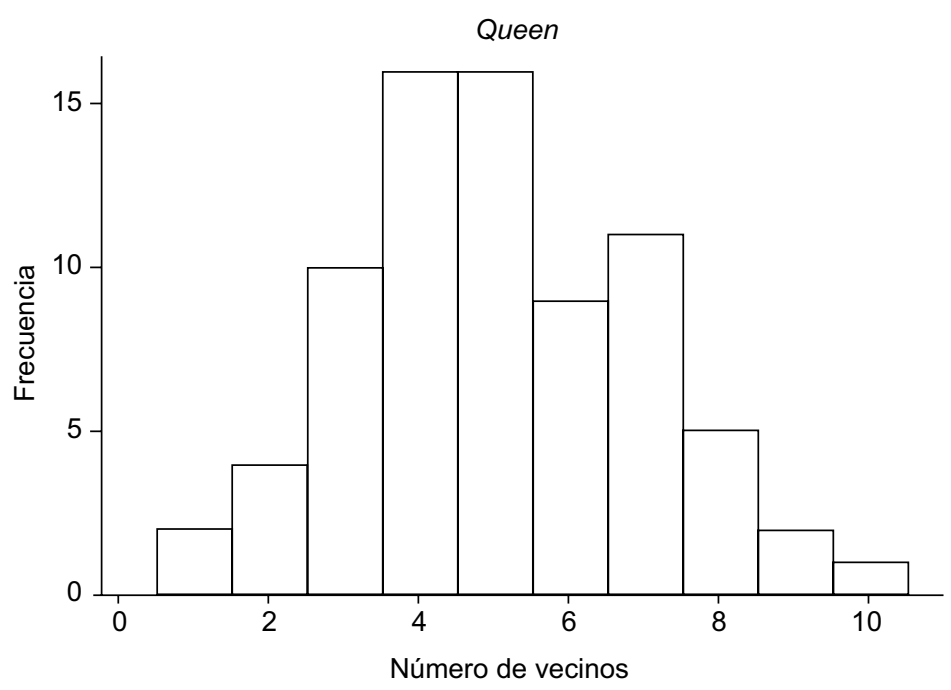

FUENTE: Elaboración propia con datos del Marco geoestadístico municipal 2005, versión 1.0. 
la ecuación 1). En este escenario consideramos los siguientes radios: $15.4 \mathrm{~km}, 30.8 \mathrm{~km}, 61.5 \mathrm{~km}$ y $92.3 \mathrm{~km}$. También se incluye un radio de $121.7 \mathrm{~km}$ cuya particularidad es generar interacción global entre todos los muncipios-delegaciones. En la gráfica 3 se presentan las distancias, el número promedio de vecinos por cada radio de interacción, así como sus histogramas de frecuencias. La lógica de la distribución de los vecindarios es simple: mientras más amplio sea el radio, más se incrementará el número de municipios que interactúa con otros, lo cual repercutirá en los procesos de actualización de la tasas de crecimiento. La amplitud de los radios está condicionada básicamente a la estructura territorial de la ZMCM; por ejemplo, en un radio de $92.3 \mathrm{~km}$ hay 50 municipios que interactúan con todos (véase en la gráfica 3 el diagrama de la parte inferior izquierda).

\section{Tipo de actualización}

Los sistemas lógicos de las computadoras consideran el tiempo en forma discreta, de ahí que puedan actualizar los estados de los agentes en formas sincrónica o asincrónica. En la literatura (Huberman y Glance, 1993; Gilbert, 2008; Izquierdo et al., 2009) se reconoce que el tipo de actualización condiciona los resultados de los modelos. En una actualización asincrónica se asigna un turno a cada agente por cada comando contenido en la programación. La mayoría de los sistemas sociales se reconocen por su naturaleza asincrónica, pues los individuos u organismos no actúan de manera simultánea, ya que en general ejecutan sus acciones de forma adaptativa valiéndose de información limitada e imperfecta.

En términos computacionales este tipo de actualización asincrónica puede presentar dos variantes: a) Ejecución asincrónica secuencial: cada agente se invoca en un orden preestablecido de acuerdo con alguna de sus características (por ejemplo, su tamaño económico o posición geográfica) y b) Ejecución asincrónica aleatoria: en cada ciclo cada agente se invoca en un orden aleatorio diferente.

El uso de la ejecución asincrónica tiene sus desventajas, pues el orden de actualización de los agentes puede influir notablemente en los resultados finales, y por lo tanto una sola corrida no es suficiente para entender la dinámica del sistema (Gilbert, 2008). Sin embargo esta desventaja puede controlarse construyendo distribuciones empíricas de resultados de $n$ simulaciones, como veremos más adelante. 
En el contexto del modelo presentado cada municipio toma un turno en el cual actualiza su estado, de modo que cuando el último municipio ejecute la regla de transición consistente en asumir como propia la tasa promedio de sus vecinos (véanse las ecuaciones 1 y 1.A), su estado no sólo será producto de las condiciones iniciales de sus vecinos, sino también de los estados previos de los municipios-delegaciones que actualizaron su estado previamente.

En un caso sincrónico se modela una situación en que los municipios actualizan su estado de manera "simultánea"; por ejemplo, una economía hipotética donde los procesos de producción, transacción y consumo se generan en forma paralela. Esto significa, en términos de simulación, que todas las entradas de los agentes se han completado antes que las salidas. Este tipo de actualización se modela para captar las dinámicas globales de los procesos de interacción espacial cuando se analizan procesos que se representan por medio de ecuaciones en diferencia (Huberman y Glance, 1993).

\section{El equilibrio del sistema y el parámetro de intensidad de interacción espacial}

El parámetro $\beta_{g}$ en las ecuaciones 1 y 1 .A mide la intensidad de la interacción espacial que existe entre las unidades regionales en las condiciones de equilibrio desarrolladas por el sistema. Independientemente del valor que $\beta_{g}$ asuma, el sistema dinámico bajo estudio genera una perfecta imitación o contagio como equilibrio del sistema; sin embargo el tipo de contagio es diferenciado, y dependiendo del valor del parámetro $\beta_{g}$ se generan los siguientes tres equilibrios de contagio: 1) cuando $\beta_{g}=1$ todas las unidades regionales $i$ adoptan la tasa promedio de crecimiento; 2) cuando $0 \leq \beta_{g}<1$, la tasa de crecimiento de $i$ tiende a cero y por lo tanto el crecimiento promedio de la ZMCM (véase la ecuación 2) también tiende a cero.

En términos formales:

$$
\begin{aligned}
\text { Si } 0 \leq \beta_{g}<1 \quad & \operatorname{Lim}_{t \rightarrow \infty} G r_{i}^{t+1} \approx 0 \\
& \lim _{t \rightarrow \infty} G r^{t+1^{*}} \approx 0
\end{aligned}
$$

Y finalmente tenemos el tercer equilibrio de contagio, cuando $\beta_{g}>1$ es fácil inferir que el crecimiento promedio de los municipios será explosivo. 


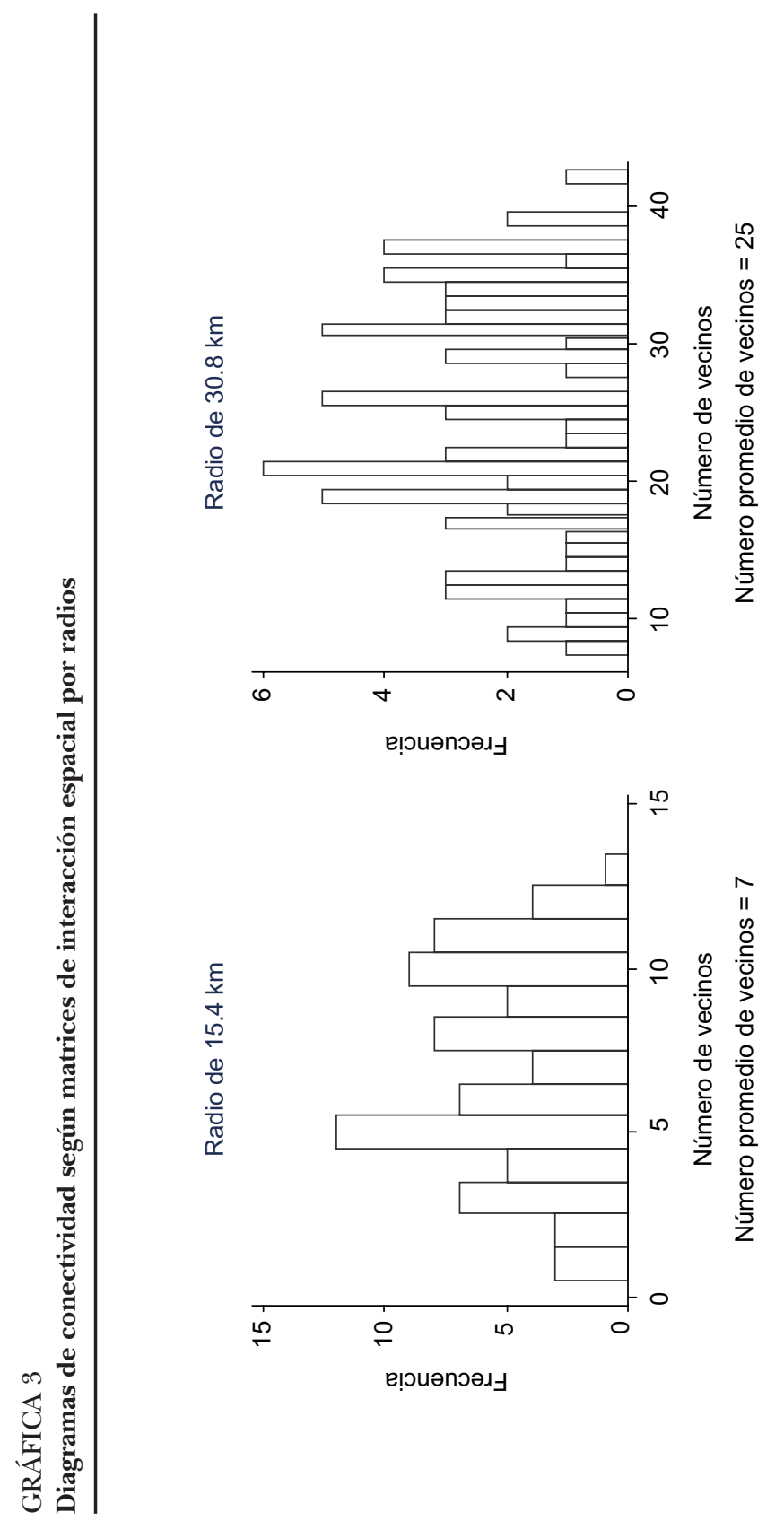




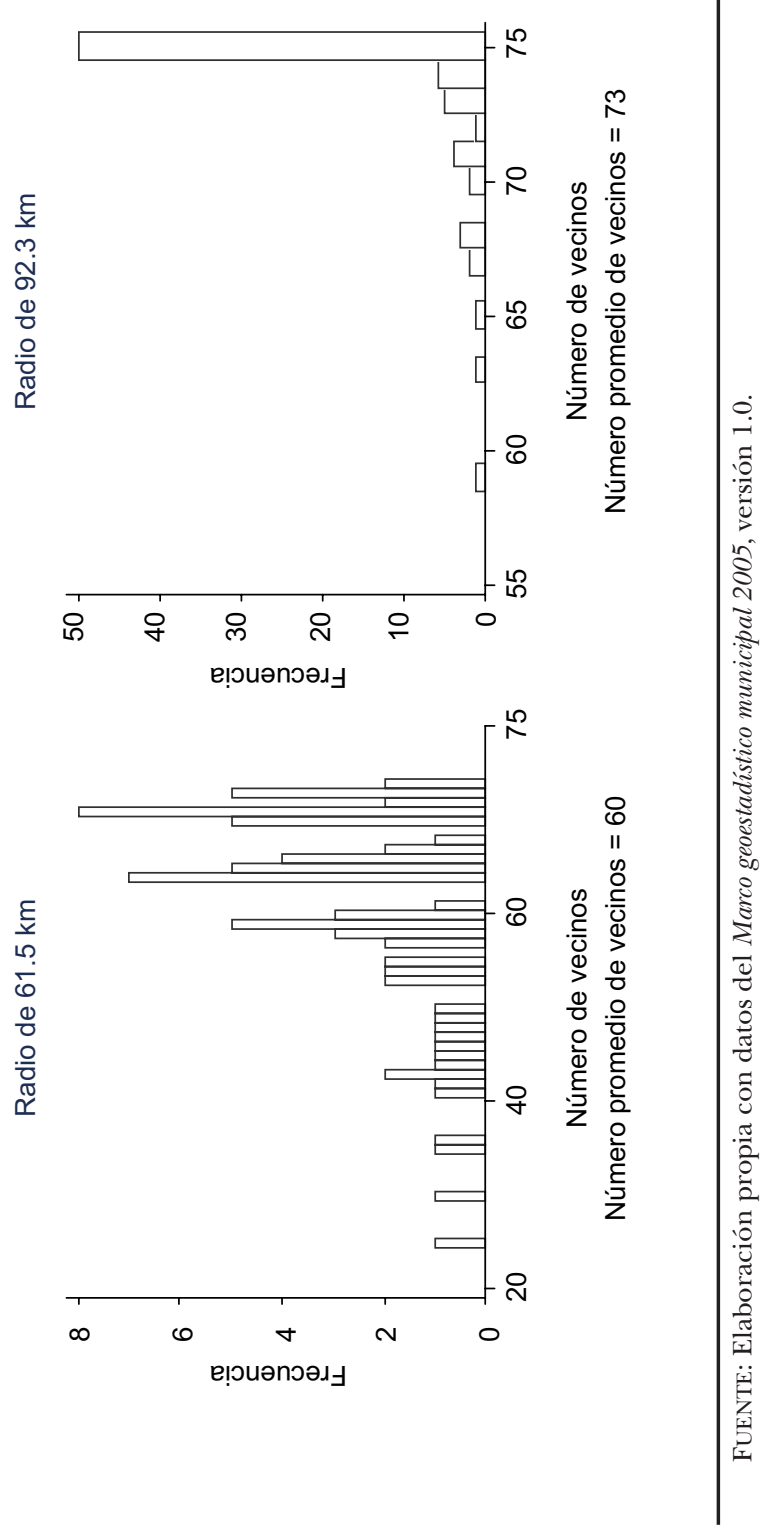


Por lo tanto, si

$$
\begin{aligned}
& \lim _{t \rightarrow \infty} G r_{i}^{t+1} \approx+\infty \\
& \beta_{g}>1 \\
& \lim _{t \rightarrow \infty} G r^{t+1^{*}} \approx+\infty
\end{aligned}
$$

Aunque este sistema dinámico de contagio parece muy simple, es capaz de desarrollar resultados complejos e interesantes bajo ciertas condiciones de simulación, en particular cuando $\beta_{g}=1$ y es muy útil para explorar dinámicas de interacción espacial entre unidades regionales reales como los municipios y delegaciones de la ZMCM. ${ }^{12}$ En la siguiente sección mostraremos algunos resultados bajo este escenario.

\section{Resultados de las simulaciones}

Aquí presentamos los resultados de la simulación atendiendo básicamente a los tres componentes del modelo que destacamos en las secciones anteriores: 1) vecindad, 2) tipo de actualización y 3) intensidad del parámetro de interacción espacial $\beta_{g}$. El propósito de la simulación es identificar las regularidades empíricas y, en su caso, los resultados que pueden ser de interés para el diseño de políticas urbanas y regionales en la ZMCM.

Iniciamos los ejercicios de simulación con tres experimentos. En el primero comparamos los resultados de una ejecución de tipo sincrónico, para lo cual evaluamos las tasas de crecimiento observadas (1998-2008) del PIB y del PIB per cápita ${ }^{13}$ contra los resultados de una ejecución sincrónica de tipo aleatorio; en este último caso usamos tasas de crecimiento del PIB generadas en forma aleatoria ${ }^{14}$ cuyos momentos se tomaron de las tasas de crecimiento observadas en el periodo 19982008 (véase el cuadro 2).

12 Cabe mencionar que si el modelo se adecua para introducir la interacción espacial de unidades regionales no contiguas y además distantes, el resultado de contagio no se ve alterado; sólo cambian las trayectorias de crecimiento hacia el equilibrio (contagio). La única forma en que se evita el contagio es "aislando" algunas de las unidades regionales del resto de tal manera que no interactúen con alguna de ellas.

13 Incorporamos el PIB per cápita y la productividad con la finalidad de considerar un posible efecto por sesgo de partición (véase la nota 9).

${ }_{14}$ Para una mejor referencia de la generación de modelos computacionales estocásticos véase Izquierdo et al., 2009. 
En el segundo experimento ejecutamos la simulación de forma asincrónica aleatoria y comparamos los resultados con la condición inicial o tasa de crecimiento promedio de todas las tasas de crecimiento de los municipios que conforman la ZMCM; en este caso la condición inicial corresponde a la tasa de crecimiento promedio observada del PIB municipal (5.7\%). Recordemos que el principio que sigue una actualización de tipo sincrónico es que la regla de transición (ecuación 1 o 1.A) se aplica al mismo tiempo a todos los municipios, mientras que en el caso de una ejecución asincrónica aleatoria la regla de transición se aplica al azar, municipio por municipio hasta que todos hayan actualizado su estado.

Finalmente, en el tercer experimento ejecutamos la simulación de manera asincrónica secuencial, calibramos con tasas de crecimiento del PIB, PIB per cápita, valor agregado censal bruto (VACB) y productividad (1998-2008). En un experimento con estas características el orden de actualización de los municipios se define a priori de acuerdo con algún criterio; en nuestro ejercicio definimos dicha actualización atendiendo a modelos de estructura urbana (monocéntrico y policéntrico). En los tres experimentos el parámetro de intensidad de interacción espacial es igual a 1 .

El contexto de interacción entre los municipios y las delegaciones en los experimentos está definido por las vecindades que describimos en el segundo apartado de la tercera sección. En todos los ejercicios el resultado emergente que se evalúa es la tasa de crecimiento global

CUADRO 2

Estadística descriptiva de las variables que se usaron en la simulación

\begin{tabular}{lcccc}
\hline & $\begin{array}{c}P I B \\
t=0\end{array}$ & $\begin{array}{c}\text { PIB per cápita } \\
t=0\end{array}$ & $\begin{array}{c}\text { VACB } \\
t=0\end{array}$ & $\begin{array}{c}\text { Productividad } \\
t=0\end{array}$ \\
\hline Media & 5.7 & 3.3 & 3.7 & -2.32 \\
Desviación estándar & 6.62 & 6.26 & 6.42 & -2.30 \\
Mínimo & -5.3 & -10.6 & -15.7 & -17.6 \\
Máximo & 27.1 & 23.4 & 29.3 & 13.2 \\
\hline
\end{tabular}

FUENTE: Los datos del PIB municipio-delegación son estimaciones (véase la nota 6). Los datos del VACB y la productividad se tomaron de los censos económicos de los años 1999, 2004 y 2009 (INEGI). Definimos la productividad laboral como el valor agregado censal bruto entre la población ocupada total, y el PIB per cápita como el PIB entre la población total. Las tasas corresponden a la media anual observada en 1998-2008. El VACB y la productividad laboral fueron deflactados con el índice del valor agregado nacional base 2003 . 
de la ZMCM, la cual está definida en la ecuación 2. En el cuadro 3 se resumen las condiciones de simulación descritas.

\section{Experimento I y Experimento II}

El propósito del primer experimento es comparar los resultados de una actualización sincrónica con los de una actualización sincrónica aleatoria. En una actualización sincrónica el orden en que se actualizan los municipios no importa, ya que todos tienen acceso a la información en el mismo momento: se elimina la retroalimentación, por lo que bajo esta condición siempre se producirá en $n$ simulaciones el mismo resultado de equilibrio (de la ecuación 2) si las condiciones iniciales son las mismas. Este tipo de experimento, en comparación con otros, tiene la ventaja de que permite indagar sobre la trayectoria individual de algún municipio o delegación.

En la columna 4 del cuadro 4 se presenta la tasa de equilibrio promedio que se obtuvo con diferentes vecindades (véanse las hileras) cuando la condición inicial es la tasa de crecimiento promedio anual del PIB o la del PIB per cápita observadas en el periodo 1998-2008. Cabe advertir que la tasa de crecimiento promedio se incrementa con el criterio de vecindad, empezando con una tasa de 4.9 para el PIB y una tasa de 2.5 para el PIB per cápita, con una vecindad queen o de radio de $15.4 \mathrm{~km}$, hasta alcanzar una tasa de crecimiento máxima de 5.7 y 3.3 respectivamente, con un radio de interacción de 121.7 km. Estas últimas tasas de equilibrio (5.7) y (3.3) coinciden con la tasa de crecimiento promedio inicial, pero ahora son una tasa de contagio o de convergencia ya que todos los municipios y delegaciones la "adoptan" cuando la ZMCM está en equilibrio. El mensaje de esta primera simulación indica que entre más local sea la interacción, menor será la tasa de convergencia o contagio respecto a la tasa de crecimiento global inicial. ${ }^{15}$

En la segunda columna del cuadro 4 se presenta el índice de Moran del crecimiento del PIB observado (1998-2008) y en la columna 3 su respectivo pseudo valor $p$ con el que podemos probar la hipótesis nula de no autocorrelación espacial. Las estadísticas indican que hay evidencia de autocorrelación espacial con el criterio de vecindad queen

${ }^{15}$ Asimismo se observa que la dinámica de contagio (y sus resultados) no se ve modificada por la variable que se utiliza (PIB o PIB per cápita), por lo que no se detecta un sesgo en los resultados debido a la partición regional escogida (municipio-delegación). 


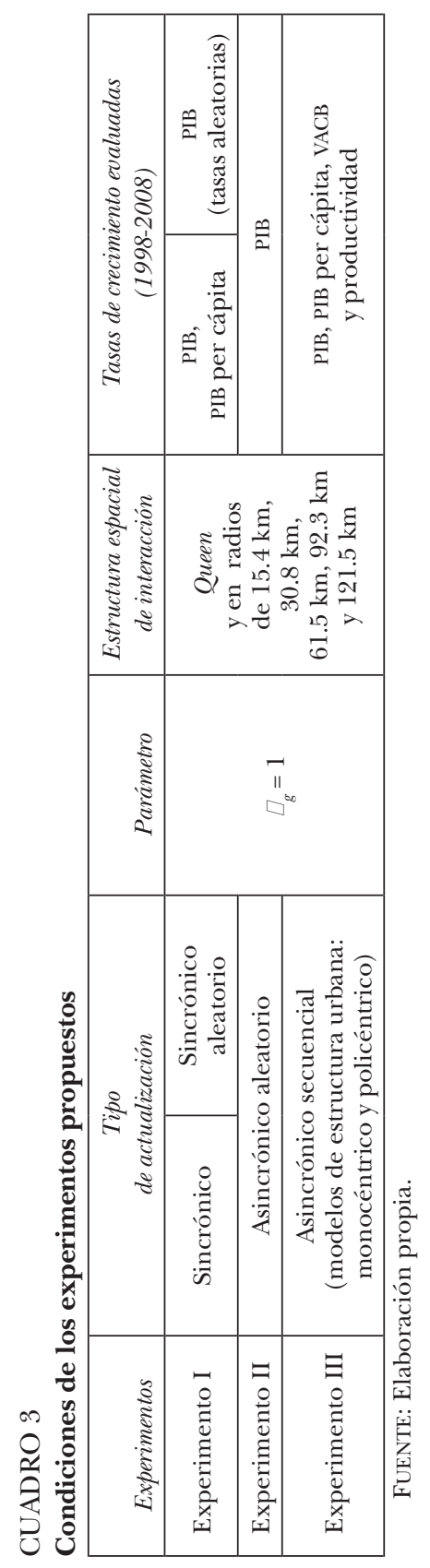


y con un radio de $15.4 \mathrm{~km}$, pero es precisamente en estos niveles bajos de conectividad donde se alcanzan las menores tasas de equilibrio de contagio. Esto indica, como veremos más adelante, que la concentración espacial observada de la tasa de crecimiento del PIB en la ZMCM (1998-2008) es la causa por la cual se obtiene, bajo un modelo de estricto contagio con condiciones de interacción local, una tasa "subóptima" de convergencia.

Ahora analizaremos las trayectorias individuales de la tasa de crecimiento del PIB de un municipio y una delegación. ${ }^{16}$ Escogimos a Ecatzingo porque es el municipio más alejado del centro de la Ciudad de México, y a Cuauhtémoc porque es la delegación que contiene a dicho centro. Sus tasas medias anuales de crecimiento (1998-2008) del PIB fueron de $9.07 \%$ (para Ecatzingo) y de $3.2 \%$ para Cuauhtémoc. En el ejercicio se utilizaron dos tipos de interacción: queen y con un radio de interacción de 15.4 km. En la gráfica 4 mostramos las trayectorias del municipio y de la delegación en una ejecución tipo sincrónico (véase la ecuación 2); asimismo la gráfica muestra claramente el dato estacionario o de equilibrio de la tasa de crecimiento.

En el cuadro 4, columna 4, se observa que la tasa de crecimiento global que se alcanza bajo una interacción queen y en un radio de $15.4 \mathrm{~km}$ es la misma (4.9\%); sin embargo las trayectorias individuales difieren y sólo Ecatzingo $(15.4 \mathrm{~km})$ alcanza el punto de equilibrio en pocos instantes, mientras que Ecatzingo (queen) sigue oscilando hasta estabilizarse en el tiempo tick 18. Cuauhtémoc queda por debajo del punto de equilibrio en los dos contextos de interacción y sólo en el largo plazo lo alcanza (para ser precisos en el tick 70). Las diferencias en las trayectorias se deben a los diferentes vecindarios; por ejemplo, en el caso de una vecindad tipo queen, Ecatzingo sólo interactúa con Atlautla $(25.8 \%)$ y adopta en el tiempo $t+1$ esta tasa elevada, mientras que en un radio de $15.4 \mathrm{~km}$ interactúa con Atlautla (25.8\%), Ozumba $(1.28 \%)$ y Tepetlixpa $(2.42 \%)$; estas tres tasas promedian 9.8 , que inmediatamente adopta el municipio de Ecatzingo en $t+1$.

Las simulaciones anteriores tienen la desventaja de que la condición inicial siempre es la misma. Con el propósito de evaluar diferentes condiciones iniciales bajo el escenario sincrónico realizamos múltiples simulaciones generando datos artificiales de manera estocástica en cada corrida, tal como detallamos al principio de esta sección (i.e. respetando la media y la desviación estándar de los datos observados).

${ }^{16}$ El modelo de simulación está diseñado de forma tal que es posible evaluar el comportamiento individual (véase la ecuación 1.A y 1.B) y el agregado (véase la ecuación 2). 


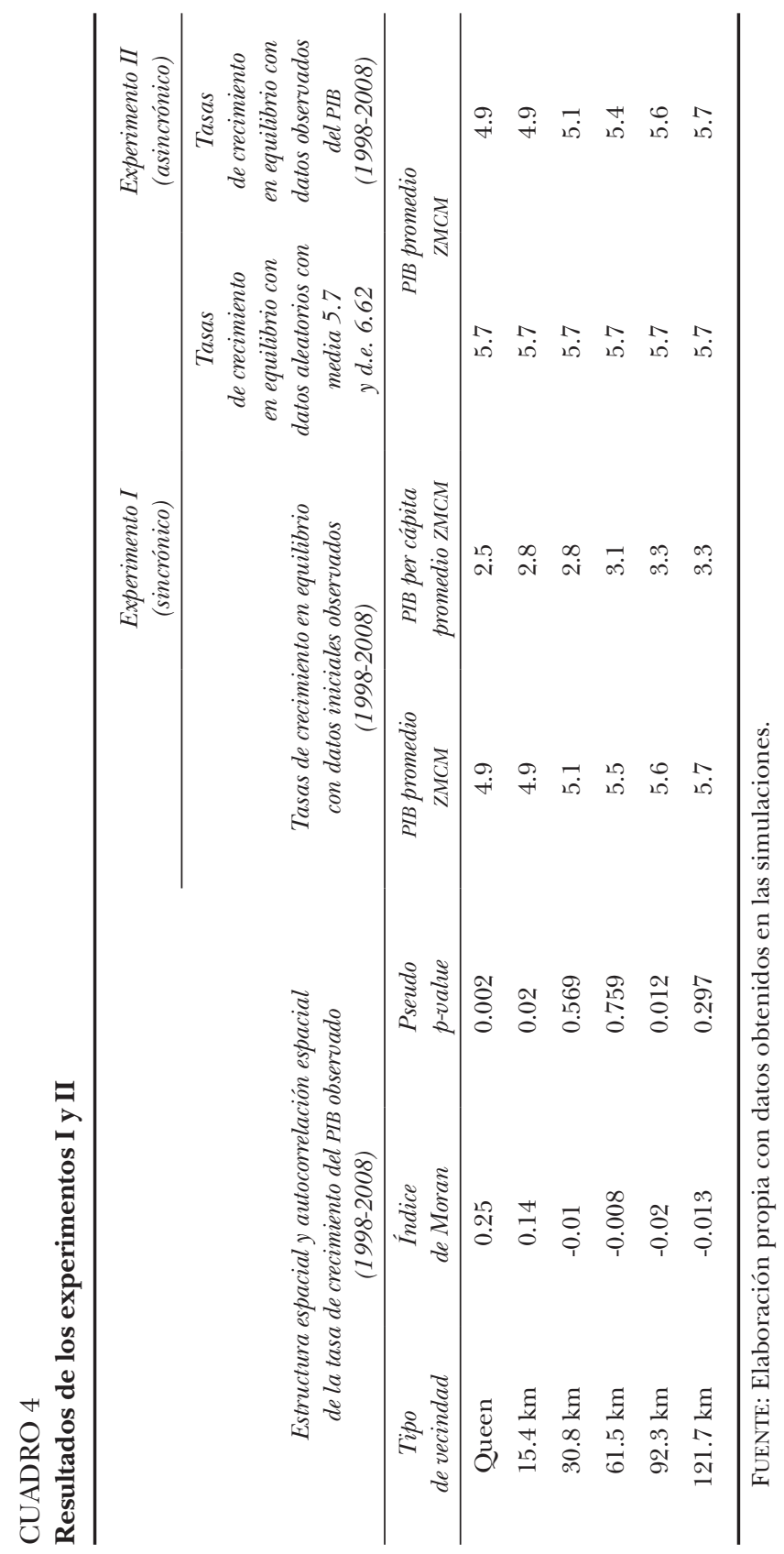




\section{GRÁFICA 4}

Trayectorias de crecimiento de Ecatzingo y Cuauhtémoc bajo una interacción espacial queen y distancia en radios de $15.4 \mathrm{~km}$

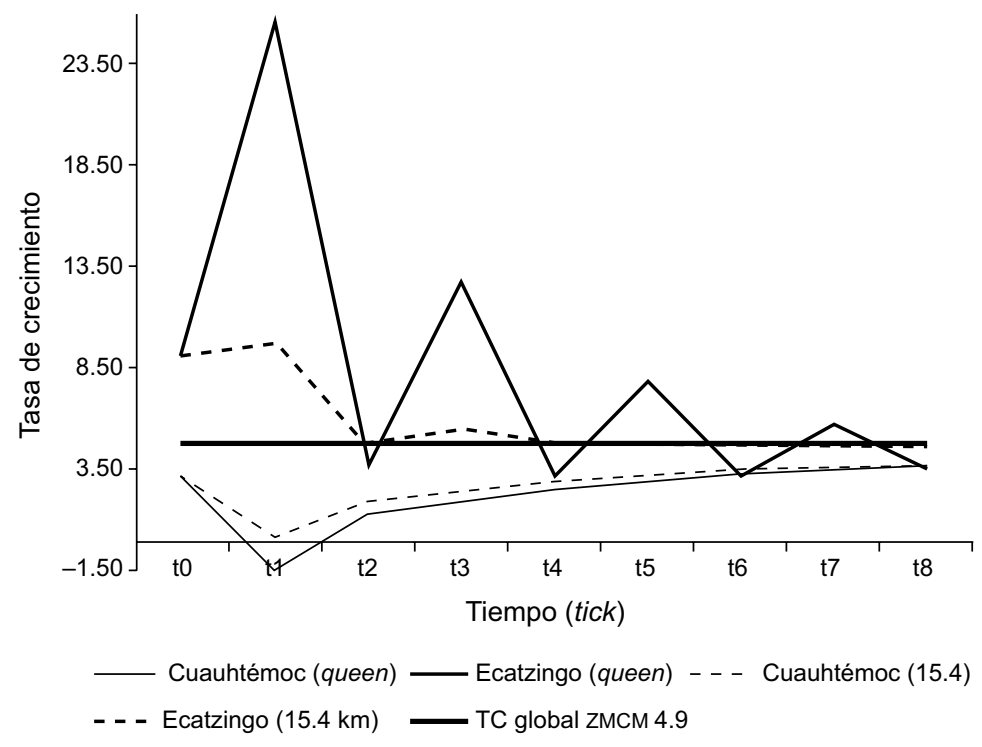

FUENTE: Elaboración propia con datos obtenidos en las simulaciones.

En concreto, se realizaron 1000 simulaciones con cada estructura espacial de interacción y se documentaron por medio de la estimación de una distribución Kernel gaussiana de las tasas de convergencia obtenidas (en equilibrio) en cada una de las corridas respectivas utilizando el parámetro de suavizado "óptimo" (véase la gráfica 5).

En la columna 6 del cuadro 4 se muestra el promedio de la distribución; se puede observar que es el mismo en cada uno de los criterios de vecindad considerados. El resultado no es del todo sorpresivo en el sentido de que las condiciones iniciales se aleatorizaron (bajo un criterio de normalidad) y los equilibrios en general se atrajeron hacia la media, y esto sin importar el tipo de vecindad. Las kernels de la gráfica 5 muestran claramente este último punto; ahí se observa que las distribuciones son simétricas (incluso casi iguales a la distribución normal teórica), por lo que la probabilidad de obtener una tasa de crecimiento por encima de la observada (véase la línea vertical punteada) es 

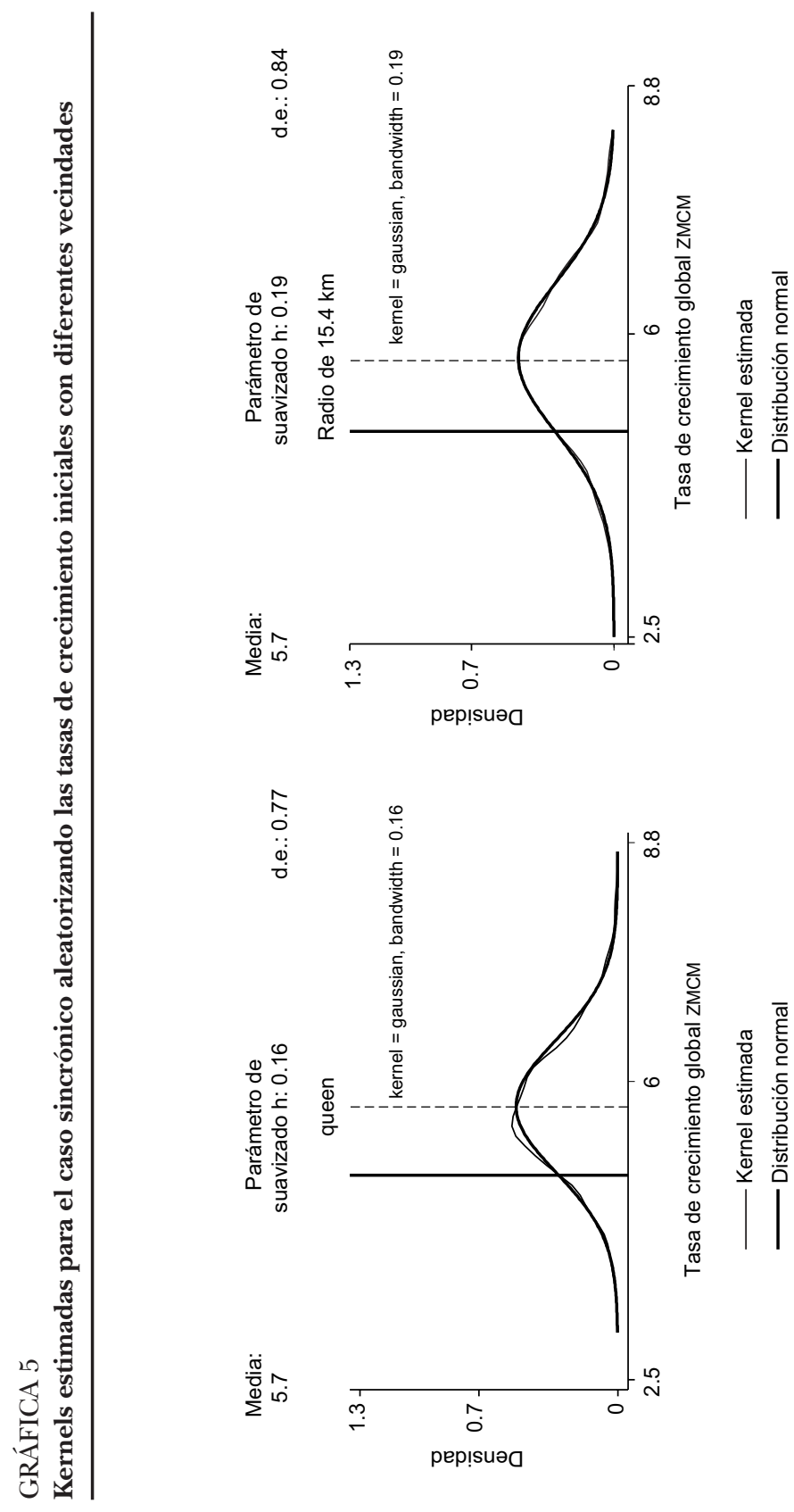

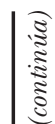
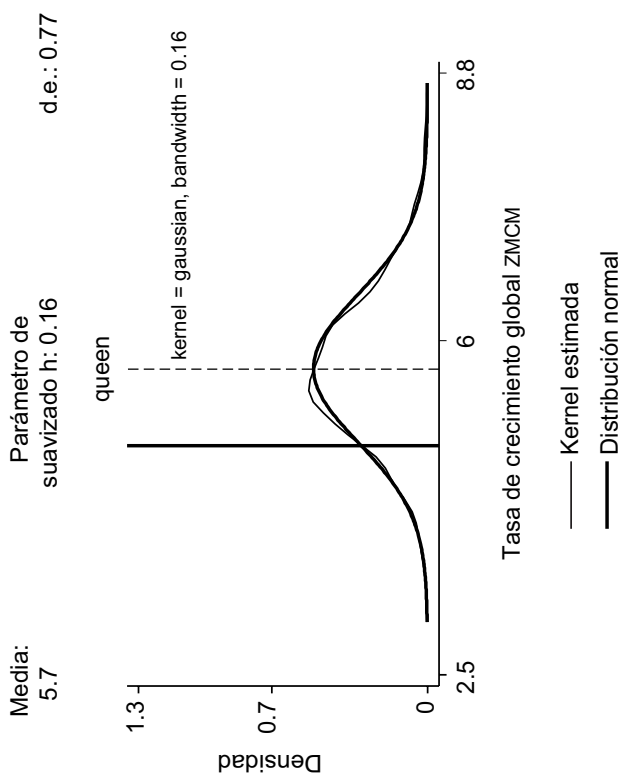


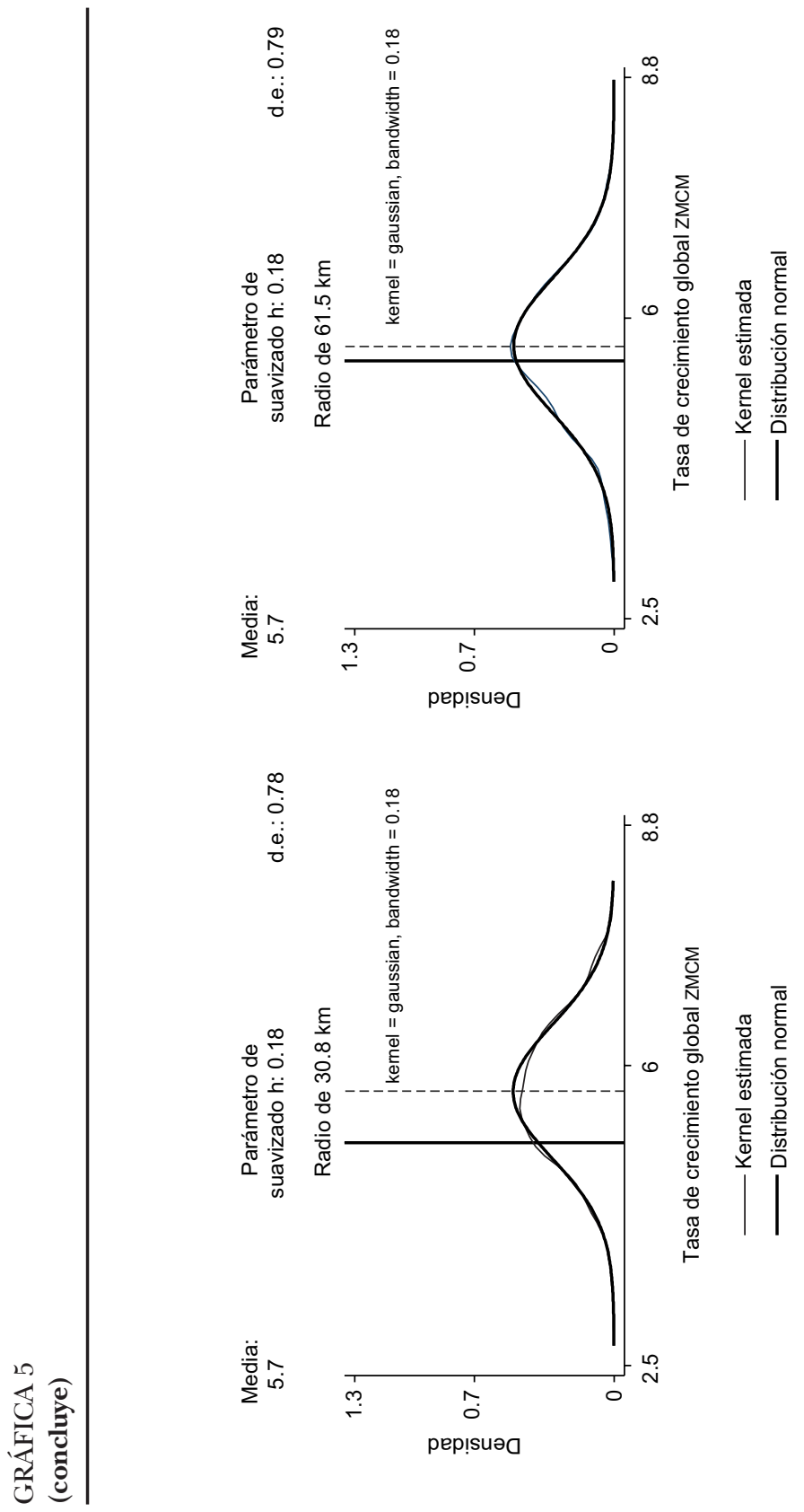




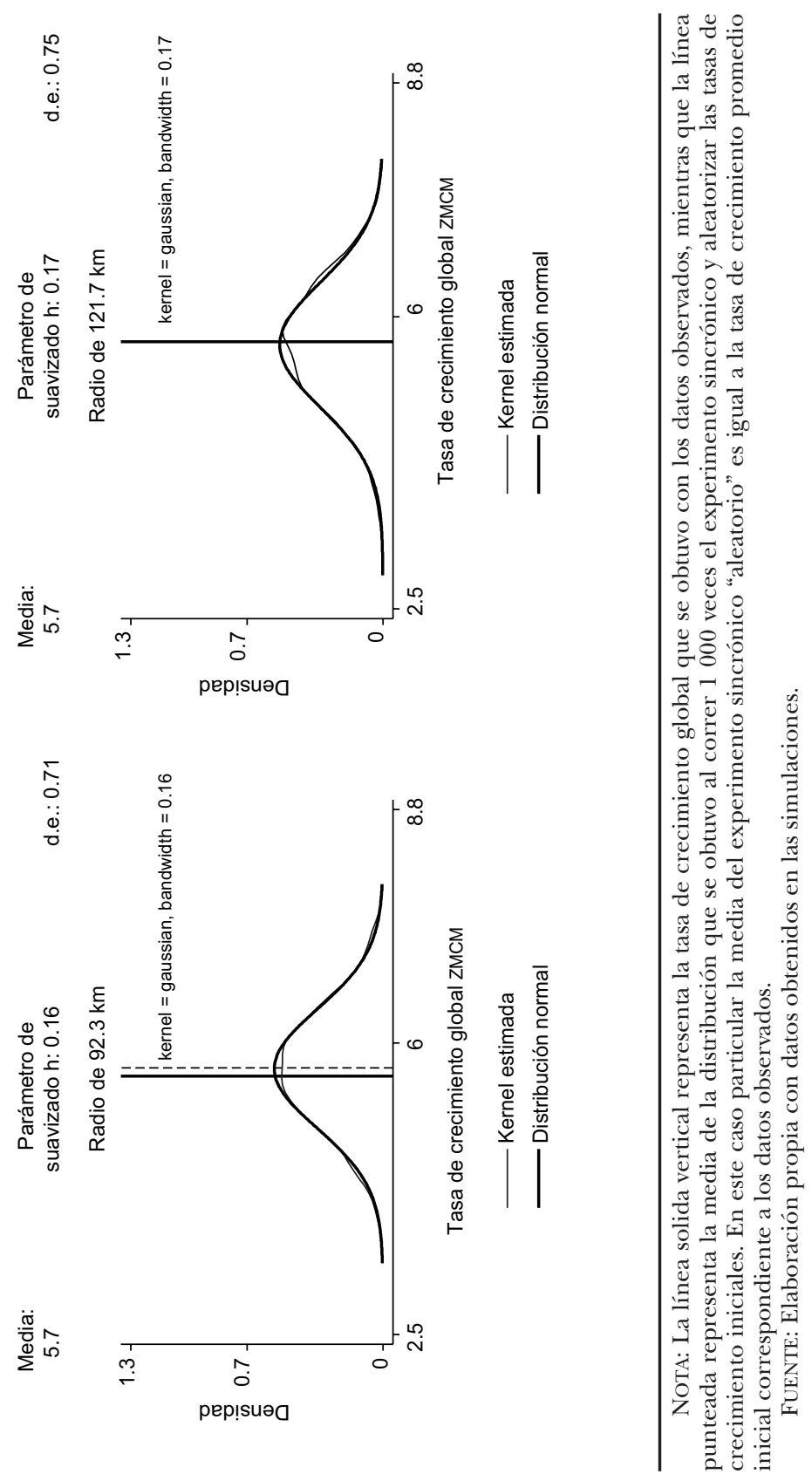


prácticamente la misma que la de una tasa de crecimiento por debajo de ésta. Esto ocurre en cada uno de los criterios de vecindad debido a que los efectos de interacción local sobre la tasa de crecimiento de equilibrio se ven cancelados en el agregado porque algunas veces hay fuerte similitud en las tasas de crecimiento local en las condiciones iniciales de arranque del modelo (como sucede con los datos observados) y en otras ocasiones no la hay.

Es interesante la comparación de los resultados anteriores con el dato que se observó para el periodo 1998-2008 (véanse las líneas verticales en las distribuciones de la gráfica 5). La tasa de convergencia promedio que se obtiene con los datos observados siempre está por debajo de la tasa promedio de convergencia de los datos aleatorios por cada tipo de vecindad, pero tiende a acercarse a la segunda cuando el criterio de interacción es mayor (incluso cuando el radio es de 121.7 las tasas son las mismas). Este resultado sólo confirma lo que habíamos ya adelantado, que la configuración de dependencia espacial que la tasa de crecimiento observada despliega en la ZMCM (véanse las columnas 2 y 3 del cuadro 4) provoca que se obtenga una tasa de equilibrio inferior (subóptima) a la esperada bajo un modelo de contagio con fuerte interacción local (queen o radio $15.4 \mathrm{~km}$ ).

Para realizar el segundo experimento, ejecución asincrónica aleatoria, se efectuaron también 1000 simulaciones con los datos observados, pero con el entendido de que el número total de posibles combinaciones de arranque es de 76. Como se expuso anteriormente, el resultado de cada corrida en este experimento es sensible a las condiciones iniciales de actualización. En términos de programación, el sistema aplica la regla de transición partiendo de la elección aleatoria de un centroide de un municipio-delegación (elige uno de 76 posibles) hasta agotar todo el conjunto; en otras palabras, en cada instante de tiempo los municipios se habrán retroalimentado de tal manera que el nuevo estado de uno afectará el estado del otro dentro de un mismo tick. Lo esencial de este orden de arranque es que en cada instante de tiempo el programa puede generar una secuencia repetida de actualización diferente; por ejemplo, si en un tick la secuencia de actualización fuera: 1, 2, 3,.., 76, sería casi nula la probabilidad que ésta se repitiera en el siguiente instante de tiempo. Con los resultados de este procedimiento se desea analizar la trayectoria de la tasa de crecimiento promedio de la ZMCM (véase la ecuación 2), comparando el valor inicial y final de equilibrio.

Para resumir los resultados de las corridas del experimento II se estimaron también kernels gaussianas de las simulaciones. En la gráfi- 
ca 6 , se muestran las distribuciones estimadas de los equilibrios que se obtuvieron conforme a los diferentes criterios de conectividad. En cada distribución se resaltan el promedio inicial global (5.7\%) (línea punteada) y la media del producto de las 1000 corridas que se generaron para cada caso (líneas verticales). También se reportan arriba de la figura respectiva el valor de la desviación estándar (d.e.) y la media de las corridas.

El primer resultado del segundo experimento que debemos resaltar es que el promedio de la distribución en casi todos los criterios de conectividad, con excepción del caso de radio de $61.5 \mathrm{~km}$ (véase la columna 7 del cuadro 4) es igual al promedio de las tasas observadas respectivas para el caso sincrónico (véase la columna 4 del cuadro 4). Sin embargo, aun bajo las condiciones del experimento II, obtenemos tasas de equilibrio subóptimas en el sentido de que la media de la distribución ahora siempre se encontrará por debajo del promedio inicial observado (5.7), salvo en el caso de la interacción global (121.7 $\mathrm{km}$ ) donde ambas tasas coinciden (véase la gráfica 7). Por lo que respecta a la forma de la distribución estimada en el experimento II podemos decir que en general emerge una estructura gaussiana y no se percibe una presencia importante de modas o de polarización; sin embargo, a diferencia de las distribuciones para el caso sincrónico aleatorizado (véase la gráfica 6), las distribuciones del experimento II tienen mayor apuntamiento, por lo que los estados de equilibrio están más centrados alrededor de la media para el caso asincrónico. En resumen, al comparar los resultados bajo la actualización asincrónica aleatoria y sincrónica "aleatoria", los resultados generales de ambos experimentos son coincidentes en el sentido de que el crecimiento de la ZMCM muestra un arreglo espacial que tiene dos efectos en el crecimiento económico (véase la gráfica 7): a) uno que desarrolla equilibrios no óptimos (por debajo del potencial) cuando el sistema opera bajo fuertes condiciones de interacción local, y $b$ ) otro que, aun bajo la presencia de una fuerte interacción cercana a la global de municipios y delegaciones (por ejemplo en radios de $61.5 \mathrm{~km}$.), genera todavía una estructura de polarización que inhibe la convergencia en las tasas de crecimiento regional (compárense la línea sólida delgada y la línea punteada de la gráfica 7). Cabe aclarar que los resultados anteriores son contingentes a la estructura espacial real de la ZMCM y a los datos del "desempeño real" que experimentaron las economías durante el periodo 1998-2008; de igual manera, hasta el momento hemos presentado los resultados en función del modelo más general de contagio. 


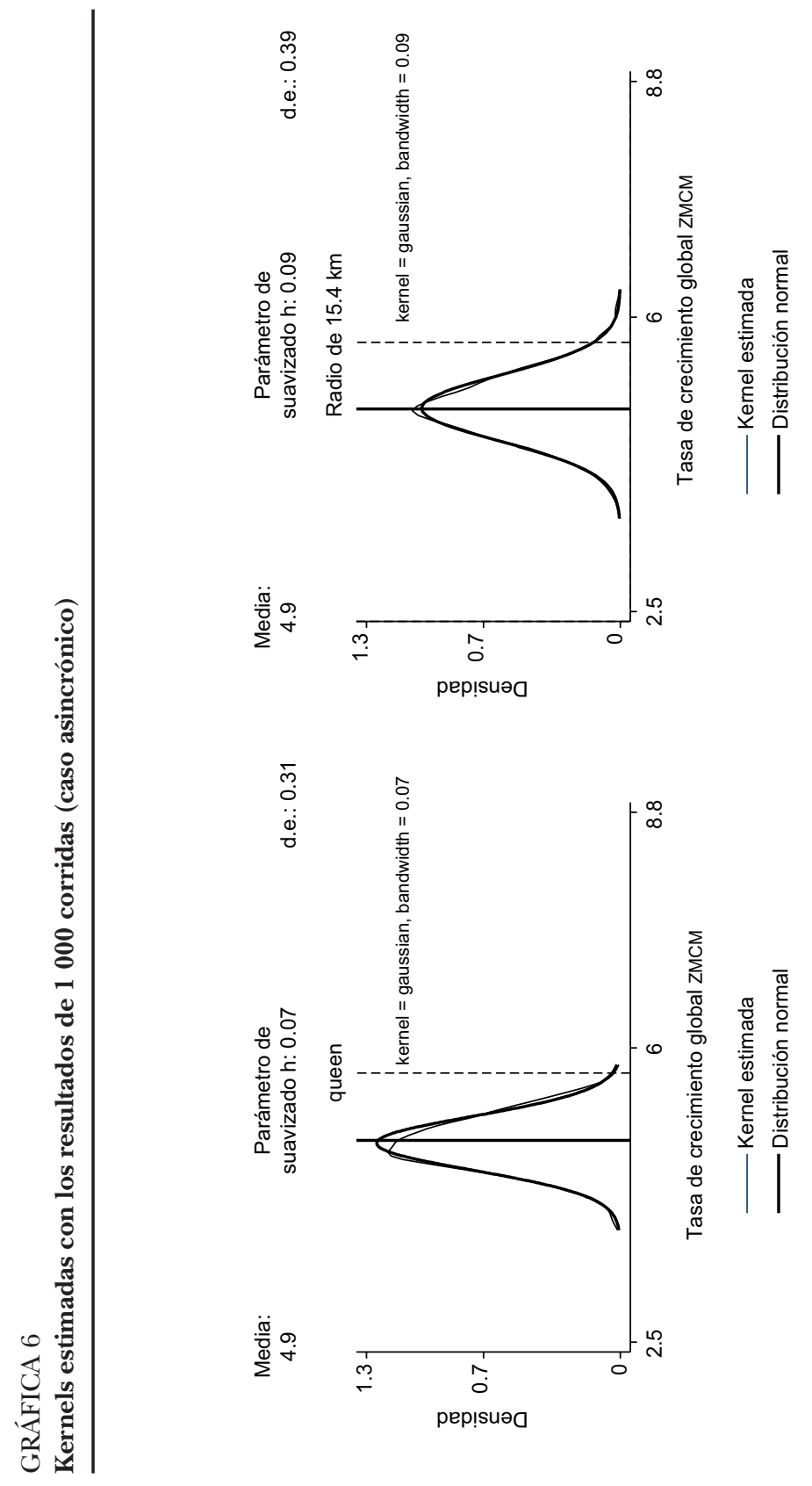




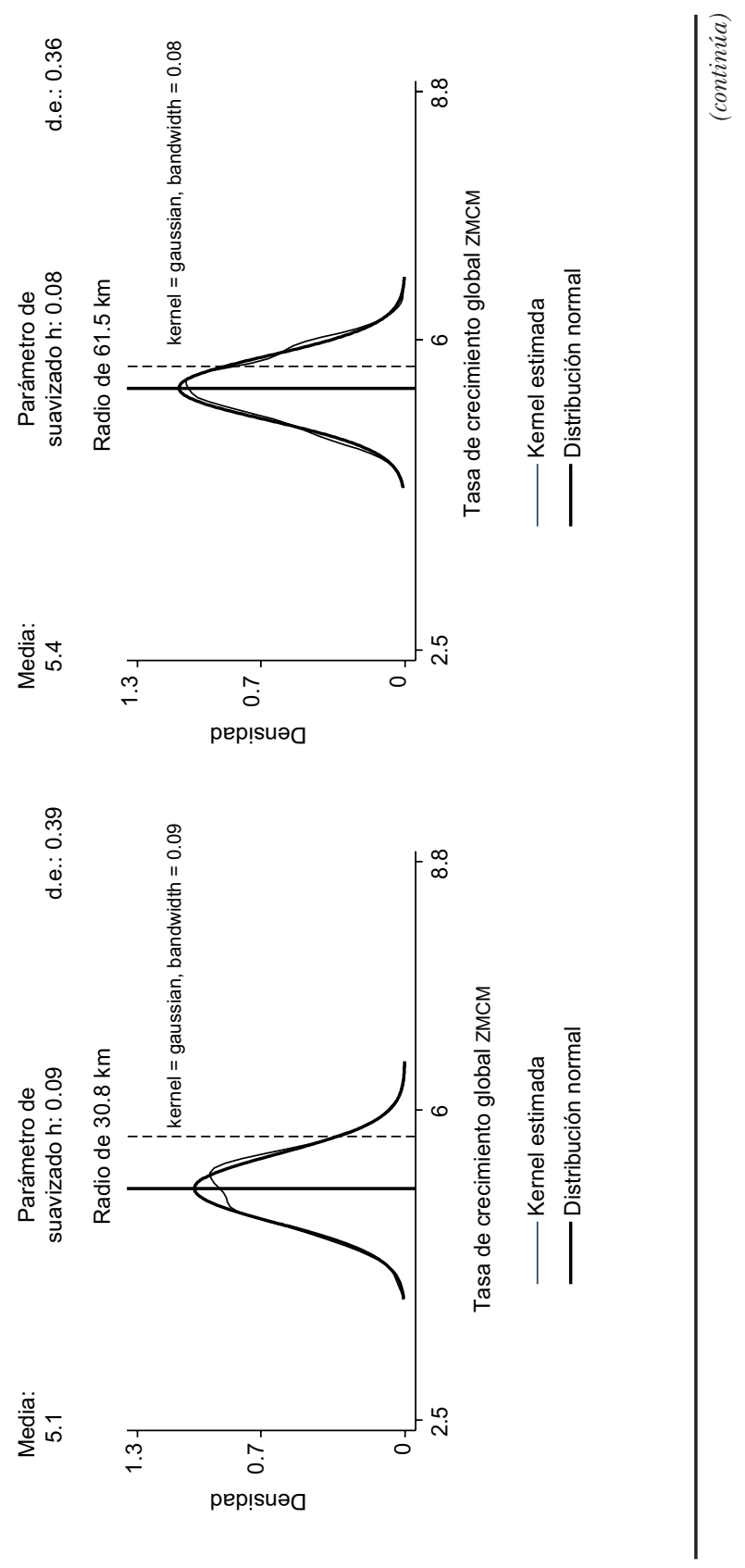




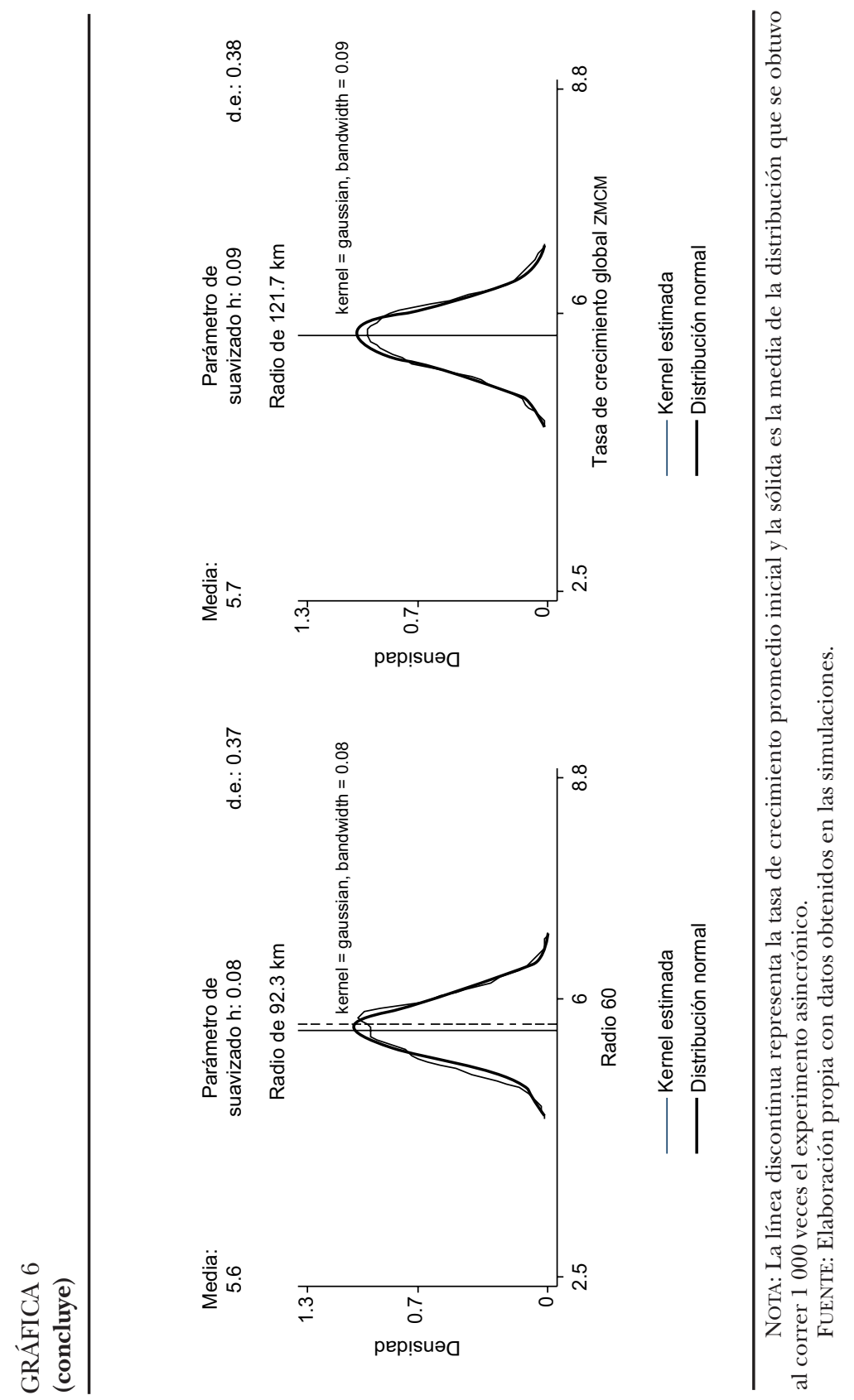




\section{GRÁFICA 7}

\section{Comparación de los resultados por tipo de actualización y vecindario}

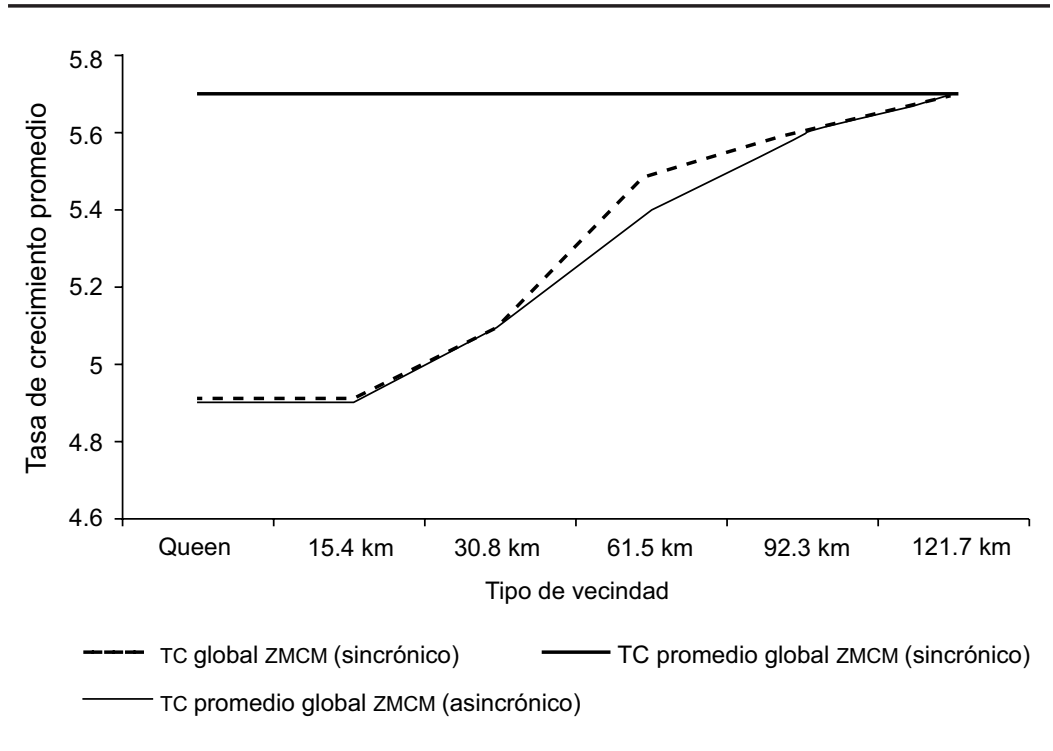

FUENTE: Elaboración propia con datos obtenidos en la simulaciones.

En la siguiente sección introduciremos el efecto de la estructura urbana sobre las tasas de crecimiento.

\section{Experimento III: inclusión de la estructura urbana de la ZMCM}

En este experimento se controló la secuencia del ciclo de acuerdo con un orden de interacción preestablecido capaz de dar cabida a estructuras espaciales económicas. Se utilizaron dos criterios: el primero se relaciona con la cercanía o lejanía hacia el centro histórico de la ZMCM (el centroide de la delegación Cuauhtémoc) y el segundo con la cercanía o lejanía de tres centros (los centroides de Cuauhtémoc, Cuajimalpa y Tlalnepantla). ${ }^{17}$ La finalidad de este ejercicio es conocer

${ }^{17}$ La definición de estos centros no es arbitraria; simplemente recoge lo que se ha discutido ampliamente en diversos trabajos sobre la estructura monocéntrica o policéntrica de la ZMCM (véase la segunda sección, nota 4). En este trabajo se consideraron como subcentros los centroides de Cuauhtémoc, Cuajimalpa y Tlalnepantla, que fueron identificados a partir de la generación de clusters mediante técnicas de autocorrelación espacial local. 
cuáles son las implicaciones de que el proceso de simulación comience "siempre" desde un mismo punto relativo a su cercanía o lejanía con el centro económico de la ciudad.

A diferencia de los experimentos I y II, en éste cada corrida llega a un único promedio, por lo que no es necesario construir distribuciones de probabilidad de los escenarios que se obtienen. Como expusimos anteriormente, el experimento incluye dos criterios de simulación que tratan de recoger las estructuras económicas del ámbito regional de la ZMCM. El primer criterio emula una estructura monocéntrica en donde el centro económico es el centroide de la delegación Cuauhtémoc; en este caso las delegaciones y los municipios irán actualizándose progresivamente de acuerdo con la distancia que exista entre los otros centroides y la delegación Cuauhtémoc. Con este criterio se pretende generar un proceso de actualización que tome en cuenta la lejanía o cercanía del centro económico. ${ }^{18}$ Por otra parte, el segundo criterio considera una estructura policéntrica, en el sentido de que los primeros en actualizar serán las delegaciones Cuauhtémoc, Cuajimalpa y Tlalnepantla, y una vez que lo hayan hecho, los siguientes serán los que se encuentren más cerca o lejos de dichos centros y así sucesivamente.

Asimismo se proponen dos casos opuestos para incorporar la distancia hacia el centro económico: uno partirá desde la o las periferias hacia el o los centros (caso A) mientras que el caso B saldrá desde el o los centros rumbo a la o las periferias. Con miras a reflexionar en términos de la generación de política regional, con el criterio monocéntrico y policéntrico se pretende simular un efecto de contagio a partir de las condiciones territoriales y de la posición que guarden respecto a los centros establecidos, ello pensando en la importancia e influencia del centro o de los centros en lo referente a las decisiones de tipo económico y político que ahí se llevan a cabo. Por ejemplo, es conocido que incluso los centros de tipo gubernamental administrativo ejercen cierta influencia en la distribución de la actividad económica de una región como la que se está modelando.

En el cuadro 5 se resumen los resultados que se obtuvieron para los criterios monocéntrico y policéntrico y sus respectivos casos A y B,

${ }^{18}$ De alguna manera en este esquema de simulación estamos incorporando los costos de transporte en la dinámica de crecimiento. Aquí se interpretaría que el sistema arranca con las unidades regionales cuyos costos de transporte son menores (las que están cercanas a los centros económicos), y hasta el final actualizan las regiones con mayores costos de transporte (las que están más alejadas de los centros económicos); o al revés, que el sistema arranque con los municipios con mayores costos de transporte y finalice con los que tienen menores costos. 
y se presentan los promedios globales que resultaron al finalizar cada corrida. En las simulaciones estamos considerando ahora la tasa de crecimiento media anual (1998-2008) de cuatro variables (véase la columna 1 del cuadro 5): PIB, PIB per cápita, valor agregado censal bruto y productividad laboral.

Observemos primero los resultados con el crecimiento del PIB y el PIB per cápita del cuadro 5. Primero se debe advertir que en general se obtienen tasas de crecimiento más altas cuando el sistema arranca de los centros económicos hacia la periferia (casos B) que cuando se empieza de la periferia hacia el centro o centros (casos A). Al contrastar un modelo monocéntrico contra uno policéntrico para el caso B (i.e., arrancando de los centros hacia la periferia) se observa que siempre hay tasas de crecimiento mayores para el caso monocéntrico en casi todas las distancias que se consideran ${ }^{19} \mathrm{y}$ para las dos variables que se incluyen (véase en la gráfica 8 las series con la etiqueta "centro-periferia"); pero cuando se parte de la periferia hacia el centro o centros (casos A) en general el escenario policéntrico genera mayores tasas de crecimiento promedio (véase en la gráfica 8 las series con etiqueta "periferia-centro").

Si consideramos el crecimiento del valor agregado censal bruto (1998-2008), en el cuadro 5 se observa que el caso B (que parte de los centros hacia la periferia) produce mejores tasas de crecimiento que el caso A (que va de la periferia hacia el centro o centros). Pero a diferencia de las variables PIB y PIB per cápita, el B tiende a ser más favorable para el caso policéntrico en el crecimiento del VACB y el A más favorable para el caso monocéntrico.

$\mathrm{Al}$ analizar la productividad (véase la gráfica 9 ) $^{20}$ se observa que en general las tasas de crecimiento menos desfavorables de la productividad se dan cuando el sistema también arranca del centro a la periferia (casos B) y al comparar la condición monocéntrica con la policéntrica encontramos que esta última tiende a desarrollar menos tasas desfavorables de crecimiento promedio. Un dato importante que

${ }^{19}$ Conviene mencionar que en un radio de $121.7 \mathrm{~km}$ cada municipio será vecino del resto de los municipios y delegaciones que conforman la ZMCM, lo que da lugar a una tasa de crecimiento equivalente a la tasa de crecimiento global observada; es decir, bajo interacción global el experimento III, independientemente de la estructura urbana, genera la misma tasa de equilibrio que los experimentos I y II.

${ }^{20}$ Cabe advertir que se parte de un crecimiento observado negativo en el periodo de -2.3 anual (véase el cuadro 2), el cual condiciona los resultados de la simulación porque se convierte en la tasa de equilibrio cuando todos los municipios y delegaciones interactúan (véase $\mathrm{km} 121.7$ en la gráfica 9). Independientemente de este resultado negativo es importante observar las tendencias de las series. 


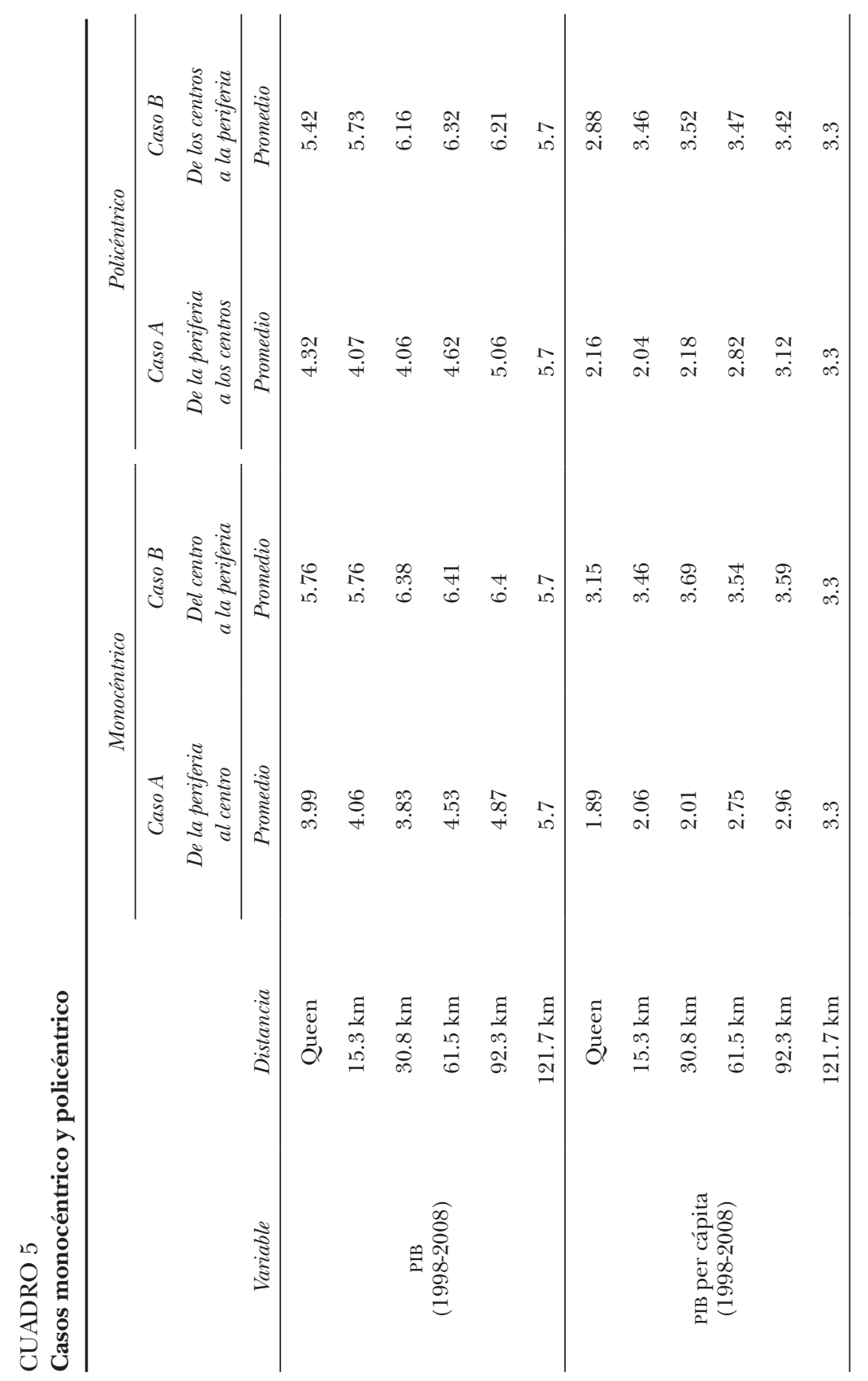




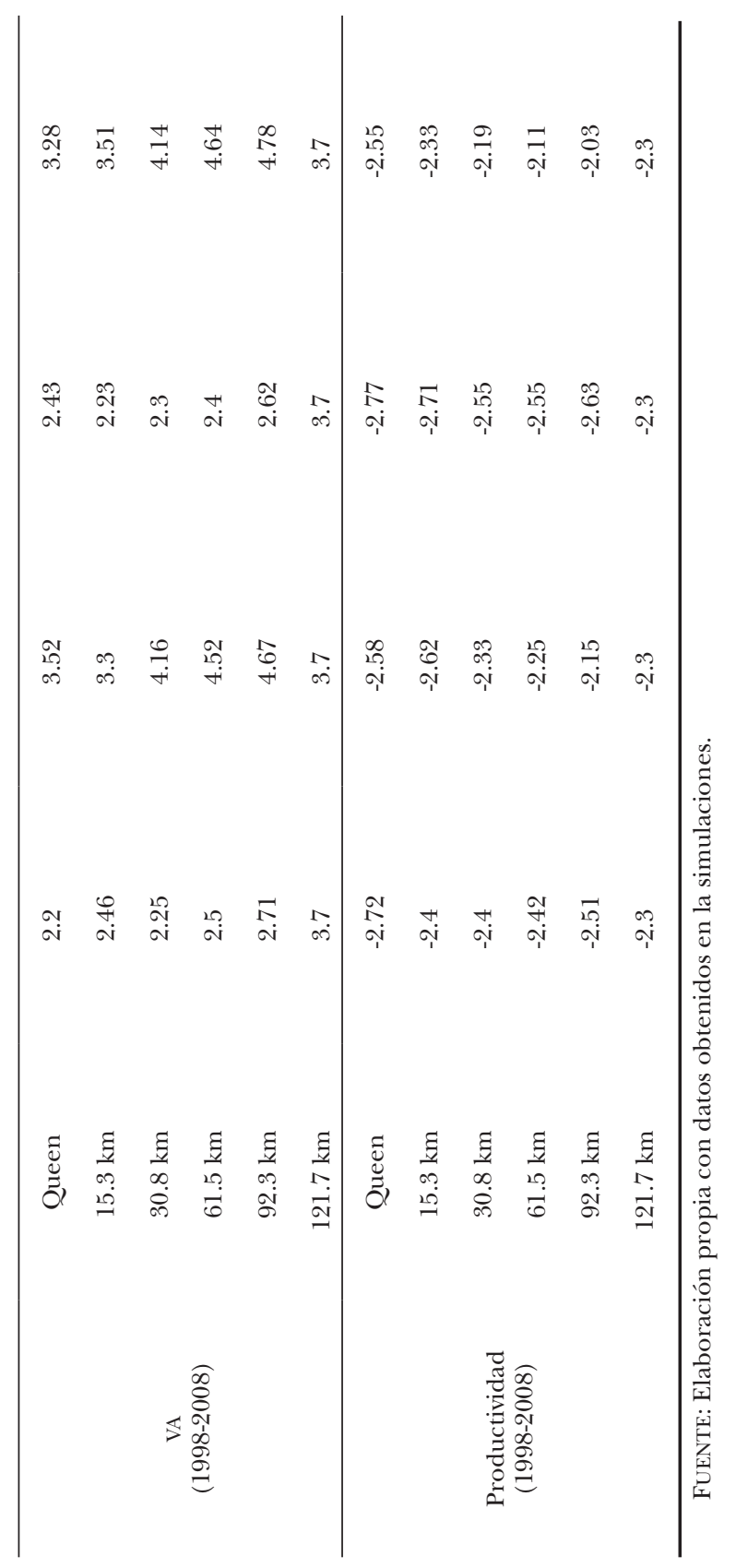




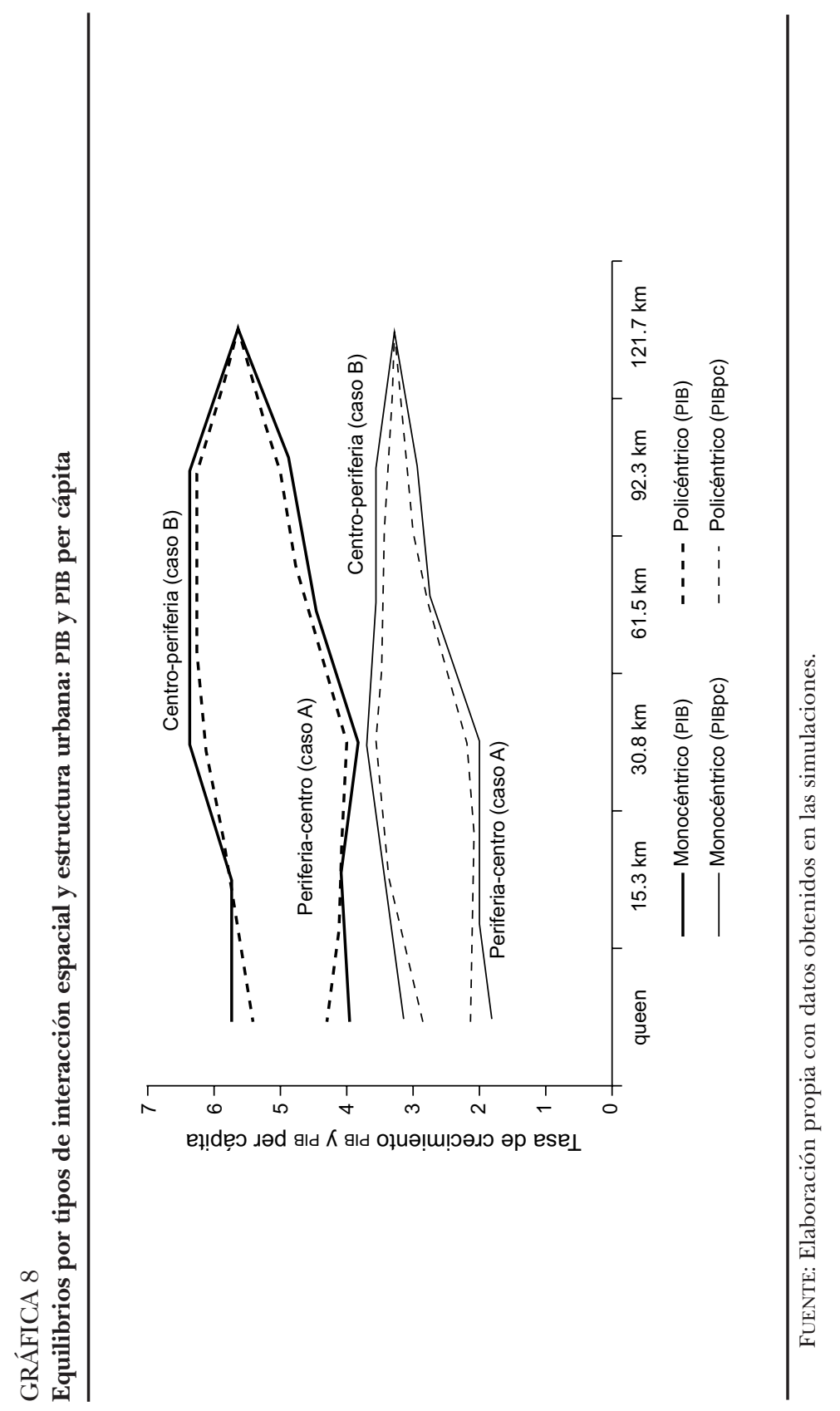


conviene advertir en la gráfica 9 es que cuando el sistema arranca de la periferia a un único centro (caso A monocéntrico), la productividad alcanza prácticamente el valor máximo posible con sólo un radio de interacción espacial de sólo 15.3 kilómetros.

Para el hecho de que el caso B genere tasas de crecimiento menos desfavorables hay una explicación sencilla: los municipios alejados de los centros económicos son los que incrementan de hecho de manera positiva su productividad laboral, y por lo tanto sugieren en principio una dinámica de catching up o convergencia respecto a los municipios más desarrollados cercanos a los centros económicos.

Finalmente mencionaremos el efecto de la distancia de interacción sobre los promedios de crecimiento de las variables representado en la gráfica 8 para los casos del PIB y del PIB per cápita y la productividad (gráfica 9). La gráfica 8 muestra que el crecimiento del PIB alcanza su máximo cuando llega a un umbral de $30.8 \mathrm{~km}$, lo cual sucede para los casos mono y policéntrico cuando el sistema arranca del centro o de los centros hacia la periferia. Es decir, no es necesario que los municipios o delegaciones interactúen con todos para que alcancen un promedio de crecimiento global máximo cuando se arranca del centro hacia la periferia, y este resultado no se modifica si la estructura urbana es mono o policéntrica. Asimismo se observa que este resultado también se replica para el caso del PIB per cápita. Sin embargo el efecto de distancia es contrario cuando se considera la variable productividad (véase la gráfica 9), pues en general cuanto mayor es la distancia, mayor será el crecimiento de la productividad. Este resultado sugiere que las derramas (o externalidades) que genera la productividad laboral son más eficientes cuando las distancias son amplias. De igual manera que en el caso del crecimiento del PIB y el PIB per cápita, los efectos de la distancia sobre el crecimiento de la productividad no parecen depender de manera importante de que la ciudad despliegue un carácter mono o policéntrico.

En general las simulaciones del experimento III indican que es importante considerar la cercanía o lejanía al centro o centros económicos en el crecimiento. En específico, la tasa de crecimiento (en equilibrio) del PIB es igual o mayor que el promedio de los últimos años si el sistema regional arranca del centro o los centros hacia la periferia, pero lo contrario ocurre cuando el sistema va de la periferia hacia el centro o centros, pues siempre el crecimiento del PIB estará por debajo del dato de equilibrio que genera la interacción global. 


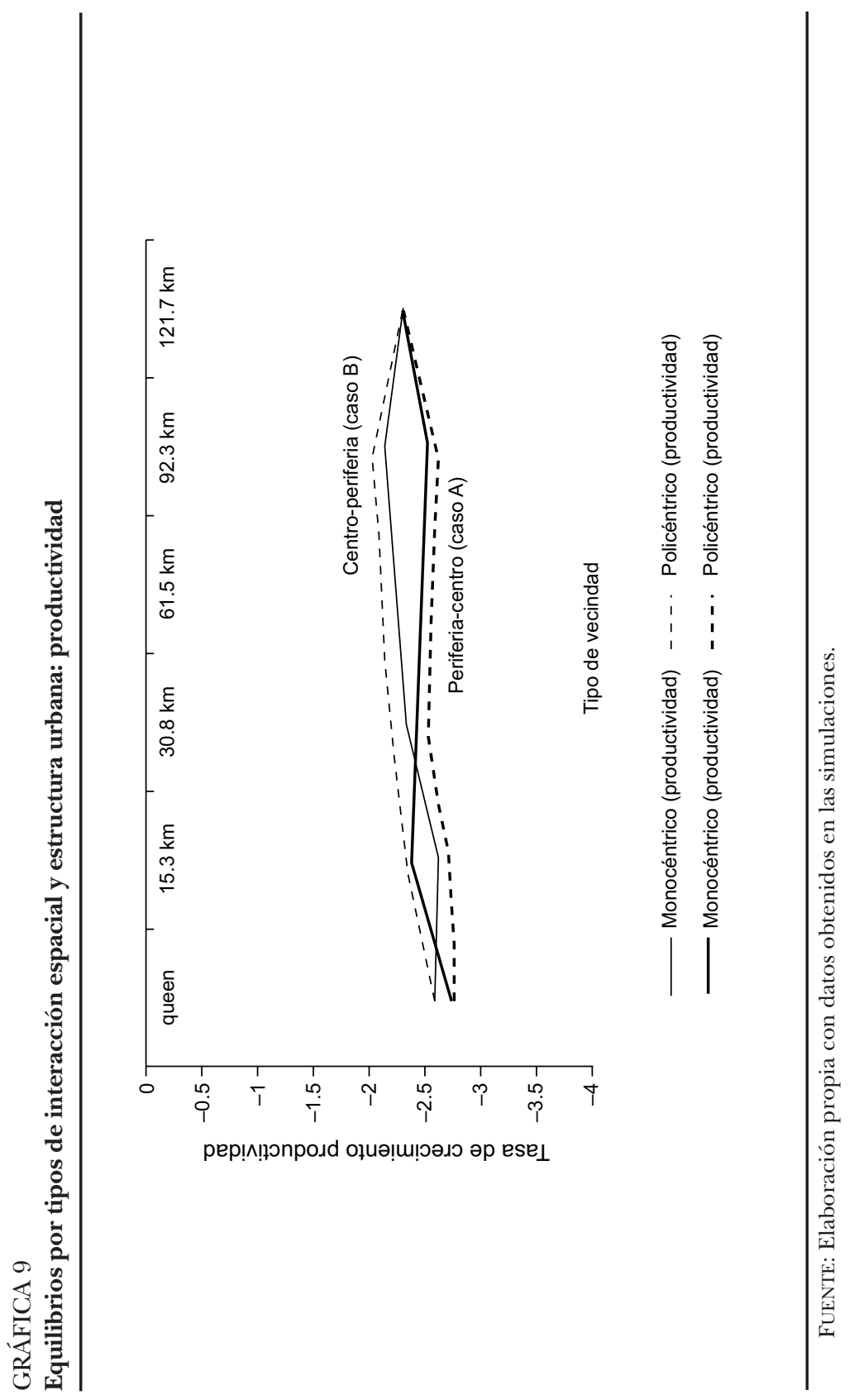


Cuando se considera el crecimiento de productividad del trabajo, en general la tasa de equilibrio es tanto mayor cuanto mayor es la distancia cuando el sistema arranca del centro a la periferia (efecto convergencia). Asimismo resalta que si el sistema arranca de la periferia al centro en el caso monocéntrico, la tasa de equilibrio prácticamente alcanza el máximo con una interacción local de $15 \mathrm{~km}$, lo que sugiere que no es necesario que los municipios o delegaciones interactúen con todos para que alcancen una tasa de crecimiento aceptable bajo esta condición monocéntrica.

Resumiendo, con las simulaciones del experimento III se muestra que es posible obtener tasas de crecimiento superiores a la observada (véase la parte derecha de las distribuciones en la gráfica 6) cuando se toma en cuenta la coordinación (asincrónica) entre municipios y delegaciones que conforman los centros o subcentros y la periferia.

\section{Conclusiones}

En este trabajo se plantea que un modelo simple de contagio entre unidades regionales como el que se propone en este ensayo puede ser un excelente método de exploración espacial de la dinámica de crecimiento económico de la ZMCM. Si bien el modelo que analizamos en este artículo es determinístico, tiene su referente en los procesos de contacto, los cuales son modelos de interacción local que han desarrollado los físicos y biólogos para analizar las propiedades espaciales de las partículas subatómicas y los microorganismos (Snyder y Nisbet, 2000). Este tipo de modelos y otros similares (como el modelo ISING) han sido adaptados de manera exitosa en las ciencias sociales por la vertiente de complejidad en economía por autores como Blume, Brock, Durlauf y Young entre otros, quienes han propuesto modelos de decisión individual con agentes interdependientes y con información limitada. Asimismo este modelo de contagio puede verse como una versión determinística de los modelos autorregresivos espaciales, que ha desarrollado la literatura de econometría espacial (Anselin, 1988) y que han sido ampliamente difundidos en los últimos años en el ámbito de los estudios urbanos y regionales.

La contribución metodológica central de este trabajo es haber integrado el modelo de contagio a un sistema de información geográfica que representa la ZMCM en los ámbitos municipal y delegacional. Tal situación no descansa per se en dar mayor realismo al modelo al 
haber incorporado la caracterización administrativo-territorial y espacial real de la ZMCM, sino en que esta vinculación entre modelo y SIG altera los resultados de equilibrio del modelo si éstos se comparan con los que se generan bajo un Autómata Celular tradicional de dos dimensiones con polígonos regulares (Page, 1999).

En este trabajo decidimos de manera deliberada explorar la dinámica de crecimiento de la ZMCM con un modelo en apariencia demasiado simple que opera sólo mediante mecanismos de contagio entre las tasas de crecimiento de las unidades regionales (municipios o delegaciones). El heterogeneizar el modelo parece un paso natural a seguir y habría sido fácil añadir otras variables (exógenas) a la regla de transición (véanse las ecuaciones 1 y 1.A) tales como el capital humano, la infraestructura urbana, la actividad sectorial, etc.; sin embargo, de haber trabajado con un modelo más complejo nos habríamos alejado del mensaje central de esta investigación en relación con los efectos que sólo la interacción local, el arreglo espacial de las unidades regionales y la estructura urbana pueden generar en la dinámica de crecimiento de la ZMCM.

Al "aislar" los efectos de contagio-difusión (externalidades) del resto de mecanismos causales nos fue posible encontrar los siguientes hallazgos del modelo que pueden ser relevantes para entender el crecimiento regional en la estructura urbana de la ZMCM. En primera instancia, las simulaciones con interacción local (sin considerar la estructura urbana) sugieren que la concentración y la dependencia espacial del crecimiento del PIB que caracterizan a la ZMCM (véase el Índice de Moran en el cuadro 4) condicionan en general la tasa de crecimiento (promedio) de equilibrio en un nivel inferior al promedio que se ha observado en los últimos años. Por el contrario, si las simulaciones se realizan bajo condiciones cercanas a la interacción global, la tasa de crecimiento (promedio) de equilibrio es muy cercana al promedio de los últimos años (escenario de convergencia). El segundo resultado a destacar es que la estructura espacial municipal-delegacional genera heterogeneidad en la dinámica de crecimiento; es decir, las trayectorias de crecimiento de los municipios o delegaciones son sensibles al tipo de vecindario o radio de distancia de interacción local que se utilice en la simulación. Un tercer resultado tiene implicaciones con relación a que la caracterización de la estructura urbana condicione o no el crecimiento local de la ZMCM; en este sentido se encontró que en general las tasas de equilibrio promedio no se ven afectadas por el carácter monocéntrico o policéntrico que pudiera asignársele a la ZMCM, pero sí por la forma en que los municipios o delegaciones interactúan con el centro 
o los subcentros económicos. En específico, el PIB y el PIB per cápita promedio crecen a mayores tasas si la dinámica regional arranca del centro a la periferia de la ZMCM, y lo contrario ocurre cuando se empieza de la periferia al centro; esto significa que el "escenario de convergencia" de una tasa de crecimiento máxima del PIB es factible con interacción local sólo bajo ciertas condiciones de coordinación entre los agentes que determinan la actividad productiva en el centro o los subcentros económicos y los municipios periféricos.

Queda para una futura investigación conferir mayor realismo geoespacial a la integración SIG del modelo (en particular, infraestructura vial y sistema de transportes), incorporar el modelo de contagio a un modelo integral de crecimiento regional, y analizar unidades regionales más desagregadas. Finalmente, quedan pendientes las implicaciones en materia de política regional (intraurbana) de este tipo de modelos para analizarlas cuidadosa y críticamente en un trabajo posterior.

\section{ANEXO}

\section{Integración a Netlogo de la cartografía de la ZMCM}

El diseño del modelo requiere información vectorial; se puede obtener el marco geoestadístico a nivel municipal en el portal de internet de INEGI <www.inegi.gob.mx>. La programación en Netlogo está constituida por tres módulos:

1) Declaración de variables

2) Ajuste inicial

3) Actualización

En el primer módulo se declara la extensión GIS y las variables globales; (cve_mpo) es la abreviación de "clave del municipio"; (nom_ mun) del nombre; y PIB, VAC y productividad se abrevian como (Gr), el número de vecinos de cada municipio (\#_vec) y la tasa de crecimiento promedio de sus vecinos como (WGr). El código es el siguiente:

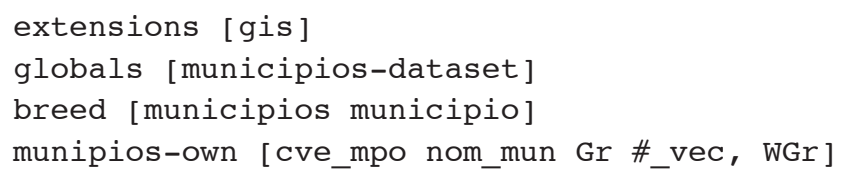


En la segunda y tercera parte se declaran dos comandos: setup y display-ZMCM. El procedimiento de la programación es el siguiente:

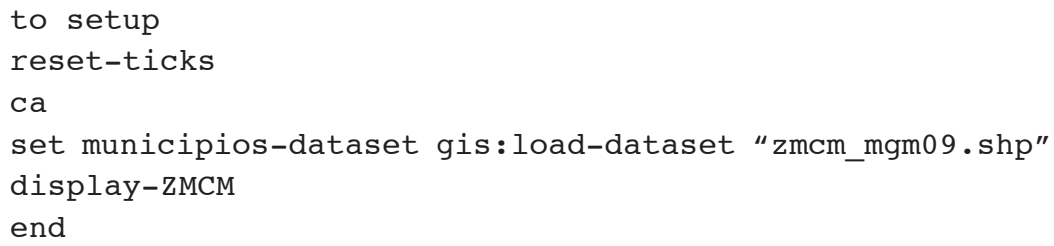

La función de setup es reiniciar a cero el conteo de ticks; eliminar cualquier resultado de corridas previas sobre la cuadrícula (ca); definir la variable global [municipios-datset] y desplegar la información vectorial correspondiente.

El comando display-ZMCM hace posible cargar una base datos espaciales con información vectorial; generar un centro de gravedad o centroide por cada municipio, el cual Netlogo reconocerá automáticamente como tortugas con forma de círculo, de color verde y con un tamaño 1, y definir los atributos correspondientes a cada agente o tortuga de acuerdo con la base de datos adjunta al archivo shp. La definición del vecindario se hace dos veces, en el comando display-ZMCM y en actualización. El código es el siguiente:

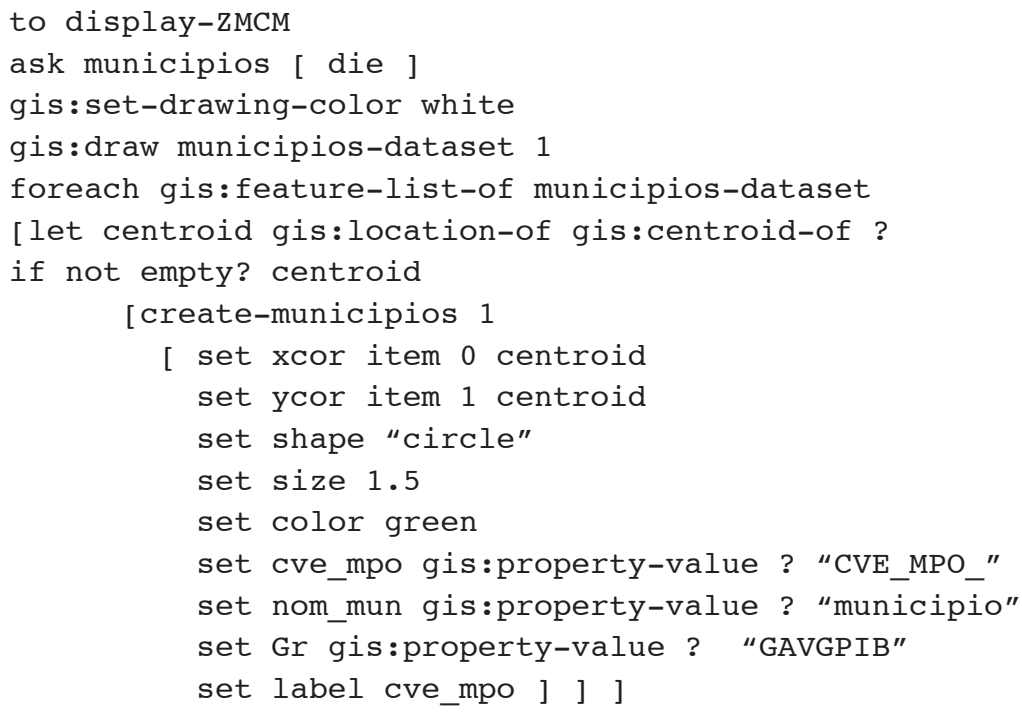




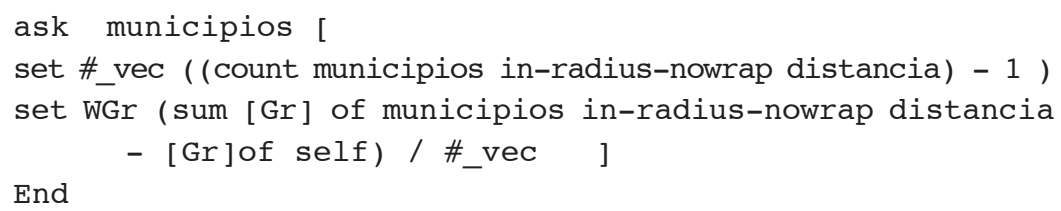

Estos comandos permiten desplegar la cartografía, los agentes y sus atributos en la cuadrícula de Netlogo. Los siguientes comandos de programación del modelo no son presentados en este anexo pero pueden ser solicitados a los autores bajo petición.

\section{Bibliografía}

Aguilar, Adrián y Concepción Alvarado (2004), "La reestructuración del espacio urbano de la Ciudad de México. ¿Hacia la metrópoli multinodal?, en Adrián Guillermo Aguilar (coord.), Procesos metropolitanos y grandes ciudades, México, Instituto de Geografía, UnAM / Miguel Ángel Porrúa, pp. 265-307.

Anselin, Luc (1988), SpatialEconometrics: Methods and Models, Dordrecht, Kluwer Academic Publishers.

Batty, Michael (2011), "Building a Science of Cities", Working Papers Series, núm. 1, Londres, UCL <http:/ / www.bartlett.ac.uk/casa/pdf/paper170.pdf>.

Brown, Daniel, Rick Riolo, Derek Robinson, Michael North y William Rand (2005), "Spatial Process and Data Models: Toward Integration of AgentBased Models and GIS", Journal of Geographical Systems, núm. 7, pp. 25-47.

Capello, Roberta y Ugo Fratesi (2008), "Space and Empirical Approaches”, en Roberta Capello y R. Camagni et al. (coords.), Modelling Regional Scenarios for the Enlarged Europe, Berlín, Springer, pp. 49-67.

Capello, Roberta y Peter Nijkam (coords.) (2009), Handbook of Regional Growth and Development Theories, Massachusetts, Edward Elgar Publishing.

Castañeda, Gonzalo (2009), "Sociomática: El estudio de los sistemas adaptables complejos en el entorno socioeconómico”, El Trimestre Económico, vol. 76, núm. 1, pp. 5-64.

Crooks, Andrew, Christian Castle y Michael Batty (2008), "Key Challenges in Agent-Based Modeling for Geo-Spatial Simulation”, Computer, Environment and Urban Systems, núm. 32, pp. 417-430.

"Declaratoria de la Zona Metropolitana del Valle de México" (2006), Gaceta Oficial del Distrito Federal, Distrito Federal, México, pp. 18-21.

Ertur, Cem y Wilfried Koch (2007), "Growth, Technological Interdependence and Saptial Externalities: Theory and Evidence", Journal of Applied Econometrics, núm. 22, pp.1033-1062. 
Escamilla, Irma (2006), “Terciarización y segregación ocupacional en la periferia expandida de la Ciudad de México", en Adrián Guillermo Aguilar (coord.), Las grandes aglomeraciones y su periferia regional, México, Instituto de Geografía, unAm / Miguel Ángel Porrúa, pp. 235-271.

Flache, Andreas y Rainer Hagselmann (2001), "Do Irregular Grids Make a Difference? Relaxing the Spatial Regularity Assumption in Cellular Models of Social Dynamics", Journal of Artificial Societies and Social Simulation, vol. 4, núm. $4<$ http://jasss.soc.surrey.ac.uk/4/4/6.html>.

Gar-On, Anthony y Li Xia (2003), "Simulation of Development Alternatives Using Neural Networks, Cellular Automata, and GIS for Urban Planning”, Photogrammetric Engineering and Remote Sensing, vol. 69, núm. 9, pp. 1043-1052.

Gilbert, Nigel (2008), Agent-Based Models, Massachusetts, Sage Publications.

Gilbert, Nigel y Klaus Troitzsch (2006), Simulación para las ciencias sociales, $2^{a}$ edición, Madrid, Mc Graw Hill.

Glaeser, Edward (2008), Cities, Agglomeration and Spatial Equilibrium, Nueva York, Oxford University Press.

Glickman, Norman (1982), "Using Empirical Models for Regional Policy Analysis", en Murat Albegov, Ake Andersson y Folke Snickars (coords.), Regional Development Modeling: Theory and Practice, Amsterdam, NorthHolland, pp. 85-104.

Graizbord, Boris y Beatriz Acuña (2004), "La estructura polinuclear del Área Metropolitana de la Ciudad de México", en Adrián Guillermo Aguilar (coord.), Procesos metropolitanos y grandes ciudades, México, Instituto de Geografía, UNAM / Miguel Ángel Porrúa, pp. 310-327.

Graizbord, Boris y Beatriz Acuña (2006), "Movilidad residencial intraurbana en la Zona Metropolitana de la Ciudad de México", en Adrián Guillermo Aguilar (coord.), Las grandes aglomeraciones y su periferia regional, México, Instituto de Geografía, UNAM / Miguel Ángel Porrúa, pp. 235-271.

Graizbord, Boris y Beatriz Acuña (2007), "Movilidad residencial en la Ciudad de México”, Estudios Demográficos y Urbanos, vol. 22, núm. 2 (65), pp. 291-235.

Hagselmann, Rainer y Andreas Flache (1998), "Understanding Complex Social Dynamics: A Plea for Cellular Automata Based Modeling”, Journal of Artificial Societies and Social Simulation, vol. 1, núm. 3 <http://www.soc.surrey. ac.uk/JASSS/1/3/1.html>.

Henderson, Vernon (2003), "The Urbanization Process and Economic Growth: The So-What Question”, Journal of Economic Growth, núm. 8, pp. 47-71.

Heppenstall, Alison, Andrew Crooks, Linda See y Michael Batty (coords.) (2012), Agent-Based Models of Geographical Systems, Nueva York, Springer.

Huberman, Bernardo y Natalie Glance (1993), "Evolutionary Games and Computer Simulation”, Proceedings of the National Academy of Sciences, núm. 90, pp. 7716-7718.

Irwin, Elena (2010), "New Directions for Urban Economic Models of Land Use: Incorporating Spatial Dynamics and Heterogeneity", Journal of Regional Science, núm. 50, pp. 65-91. 


\section{Valdivia y Linares, UN MODELO DE SIMULACIÓN}

Izquierdo, Luis, Segismundo Izquierdo, José Galán y José Santos (2009), "Techniques to Understand Computer Simulation: Markov Chain Analysis", Journal of Artificial Societies and Social Simulation, vol. 12, núm. 16 $<$ http://jasss.soc.surrey.ac.uk/12/1/6.html>.

Liggett, Thomas (1985), Interacting Particle Systems, Nueva York, Springer.

Manson, Steven (2004), "Land Use in the Southern Yucatán Peninsular Region of Mexico: Scenarios of Population and Institutional Change", Computers, Environment and Urban Systems, núm. 30, pp. 230-253.

Moreno, Niandry, Fang Wang y Danielle Marceou (2009), "Implementation of a Dynamic Neighborhood in a Land-Use Vector-Based Cellular Automata Model”, Computers, Environment and Urban Systems, núm. 33, pp. 44-54.

Page, Scott (1999), "On the Emergence of Cities", Journal of Urban Economics, núm. 45, pp. 184-208.

Park, Robert y Ernest Burgess (1925), The City, Chicago, University of Chicago Press.

Peeters, Ludo y Coro Chasco (2006), "Ecological Inference and Spatial Heterogeneity: an Entropy-Based Distributionally Weighted Regression Approach”, Papers in Regional Science, vol. 85, núm. 2, pp. 257-276.

Quigley, John (1998), "Urban Diversity and Economic Growth", Journal of Economic Perspectives, vol. 12, núm. 2, pp. 127-138.

Resende, Guilherme (2011), "Multiple Dimensions of Regional Economic Growth: The Brazilian Case, 1991-2000”, Papers in Regional Science, vol. 90, núm. 3, pp. 629-662.

Richardson, Harry (1988), "Monocentric vs. Policentric Models: The Future of Urban Economics in Regional Science”, The Annals of Regional Science, vol. 22, núm. 2, pp. 1-12.

Saraví, Gonzalo (2008), "Mundos aislados, segregación urbana y desigualdad en la Ciudad de México”, Eure, vol. 34, núm. 103, pp. 93-110.

Schelling, Thomas (1978), Micromotives and Macrobehavior, Nueva York, WW Norton.

Snyder, Robin y Roger Nisbet (2000), "Spatial Structure and Fluctuations in the Contact Process and Related Models", Bulletin of Mathematical Biology, núm. 62, pp. 959-975.

Sobrino, Jaime (2006), "Patrones de dispersión intrametropolitana en México", Estudios Demográficos y Urbanos, vol. 22, núm. 3 (66), pp. 583-617. Disponible en <biblioteca.colmex.mx/revistas $>$.

Son, Hyun Hwa (2012), "A Welfare-Based Approach to Aggregating Growth Rates across Countries" Oxford Bulletin of Economics and Statics, vol. 74, núm. 1, pp. 152-161.

Suárez, Manuel y Javier Delgado (2009), "Is Mexico City Policentric? A Trip Attraction Capacity Approach”, Urban Studies, vol. 46, núm. 10, pp. 21872211.

Suárez, Manuel y Javier Delgado (2010), "Patrones de movilidad residencial en la Ciudad de México como evidencia de co-localización de población y empleos", Eure, vol. 36, núm. 107, pp. 67-91. 


\section{Acerca de los autores}

Marcos Valdivia López es doctor en Economía por la New School for Social Research de Nueva York. Es investigador en el Programa de Estudios Regionales del Centro Regional de Investigaciones Multidisciplinarias de la UNAM y profesor en el Posgrado de Economía de la UNAM dentro del campo de conocimiento de la economía urbana y regional. Sus líneas principales de investigación se concentran en las áreas de la economía espacial y computacional, la geografía económica, la economía de las ciudades, los mercados laborales y la migración. Es miembro del Sistema Nacional de Investigadores de México, nivel I.

Nelly Esmeralda Linares Sánchez es licenciada en Economía por la Facultad de Estudios Superiores (FES) Acatlán y maestra en Economía por el posgrado en Economía de la UNAM. Es docente en la FES-Acatlán y ha participado como especialista en el área de la economía urbana y regional en diversos proyectos de investigación en la UNAM y otros organismos privados. 